\title{
Compensation method in sensor technology: a system-based description
}

\author{
V. Schulz ${ }^{1}$, G. Gerlach ${ }^{1}$, and K. Röbenack ${ }^{2}$ \\ ${ }^{1}$ Solid-State Electronics Laboratory, Technische Universität Dresden, 01062 Dresden, Germany \\ ${ }^{2}$ Institute of Control Theory, Technische Universität Dresden, 01062 Dresden, Germany
}

Correspondence to: V. Schulz (volker.schulz@mailbox.tu-dresden.de)

Received: 24 May 2012 - Revised: 17 July 2012 - Accepted: 23 July 2012 - Published: 3 August 2012

\begin{abstract}
In measurement science and engineering, the method of compensation plays a decisive role and is widely used in practical applications, in particular for sensors and measurement systems, where high accuracy is required. However, a general theoretical system description of this method with particular respect to figures of merit in sensor technology does not exist yet. Nevertheless, this is important for a real understanding of the system's structure and its properties and would facilitate prospective sensor design. Within this work, we provide a general system-based description and comparison of both the compensation and the deflection method. Based on a general sensor model and selected transfer functions, which cover most sensor types, important sensor properties like static deviations in sensitivity, long-term drift effects, response time, output signal characteristics as well as nonlinearities and hysteresis are studied in a systematic fashion for both measurement methods. In the case of a compensation method, the core sensor element is part of a controlled closed-loop system, leading to different system properties compared to an open-loop sensor operated in deflection method. The influence of linear standard controllers, which are widely used in industrial measurement and control systems, is studied with respect to the sensor properties. In the conclusions we will summarize which controller type is appropriate for the attainment of a specifically targeted sensor behavior.
\end{abstract}

1

\section{Introduction}

"The history of science is the history of measurement" (Cattell, 1893). Even though claimed by a psychologist in the late 19 th century, the validity of this statement in the fields of science and engineering is unchallenged. Measurement technology has explosively developed, and is still immensely growing, with an almost unmanageable diversity of complex sensors and measurement systems. Despite this variety and incessant new developments, the improvement of existing sensor concepts has always been of high interest. In almost every field of sensors, improvements regarding accuracy, repeatability, drift and hysteresis compensation, error proneness, response time and many more are highly desirable, regardless of the specific sensor principle. Usually, the sensor properties of a specific sensor depend on the transducer principle, the sensor material properties and the measurand. In general, sensors can be operated using different measurement meth- ods (ISO/IEC Guide 99, 2007). Nowadays, most sensors use the deflection method where the sensor output signal is a direct measure of the input signal. This method comprises the lowest demand in terms of system complexity, but on the other hand the sensor output signal is directly determined by the sensor properties, leading to a pre-determined, but often insufficient sensor performance for specific applications.

A different method known in measurement technology is the method of compensation. Possibly the most prominent example is the mechanical beam balance (Dyer, 2001, p. 302), where the beam angle is altered by the addition of a sample weight into one tray and subsequently restored by adding of the same weight to the other tray, resulting in a balanced null-deflection state of the beam. The counterweight can be determined more precisely than the beam angle, which leads to an overall higher measurement precision compared to the deflection method. In general, the compensation method can be applied to a wide range of sensors, 


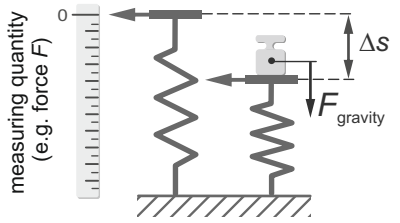

(a)

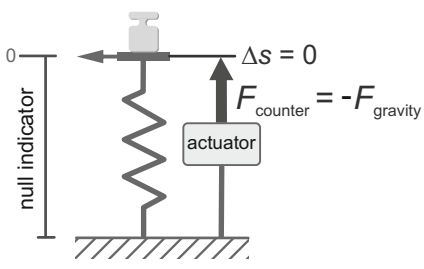

(b)
Figure 1. Simplified schematic illustration of the principle of operation of (a) the deflection method and (b) the compensation method. The spring represents an arbitrary transducer, whereas the mass of the weight generally represents the input signal.

Table 1. Elementary comparison between deflection method and compensation method in measurement.

\begin{tabular}{lll}
\hline & Deflection method & Compensation method \\
\hline System model & $X_{i} \rightarrow G_{s} \rightarrow X_{o}$ & $X_{i} \rightarrow \widetilde{G}_{s}$ \\
Input & $\begin{array}{l}\text { Measurand } X_{\mathrm{i}} \\
\text { (e.g. force } F)\end{array}$ & Measurand $X_{\mathrm{i}}$ \\
Output & $\begin{array}{l}\text { signal } X_{\mathrm{o}} \\
(\text { e.g. output voltage) })\end{array}$ & $\begin{array}{l}\text { compensation quantity } X_{\mathrm{c}} \\
\text { (e. g. force) }\end{array}$ \\
Sensitivity & $S^{\text {defl }}=\left|\frac{X_{\mathrm{o}}}{X_{\mathrm{i}}}\right|=\left|G_{\mathrm{s}}\right|$ & $S^{\text {comp }}=\left|\frac{X_{\mathrm{c}}}{X_{\mathrm{i}}}\right|=\left|\frac{\tilde{G}_{\mathrm{s}}}{1+\tilde{G}_{\mathrm{s}}}\right|$ \\
\hline
\end{tabular}

almost irrespective of the sensor principle. However, the applicability of this method implies the existence of an actuator unit (Fig. 1) which is able to physically generate the compensation quantity. In this method, the actual sensor output signal is measured, subtracted from a reference value, and the difference is fed back to the sensor input via a controller/actuator unit, until the difference between output signal and reference value reaches zero. Thus, the static sensor output signal remains constant for changes in the input signal. Nevertheless, the "force" provided by the actuator needed to maintain this balanced state is directly related to the input signal. Compared to the deflection method, which is an open-loop configuration, a sensor operated with the compensation method is a closed-loop system. This leads to different system properties solely due to the feedback structure as outlined in Table 1. Furthermore, because of the perpetuation of the balanced "undeflected" state, the sensor properties negligibly contribute to the overall system behavior, leading to smaller measurement uncertainties and hence to better measurement results. Additionally, the properties of the feedback loop can be set and tuned systematically. This is the reason why the compensation method is used in particular in technical applications with high-precision requirements or expanded operation fields, like precision balances (Krause,
2005), AFMs (in constant force mode) (Bhushan, 2005), broadband lambda probes (Bosch $\mathrm{GmbH}, 2010$ ), hot-wire anemometers (in constant temperature operation) (Fingerson and Freymuth, 1996; Tavoularis, 2005), MEMS-based accelerometers (Che and Oh, 1996; Stuart-Watson and Tapson, 2004), continous non-invasive blood pressure monitoring (Fortina et al., 2006), or even in hydrogel-based sensors very recently (Schulz et al., 2011) (see Sect. 2). Despite the potential benefits of the compensation method in current and future measurement technology, surprisingly, no general theoretical description of the properties of a sensor operated with the compensation method is available. In particular, theoretical considerations concerning the properties of such sensors with respect to particular requirements in sensor technology like high sensitivity, fast response time, robustness against long-term instabilities of the core sensor element (see $g_{\mathrm{s}}$ in Fig. 6) do not exist to our knowledge. Mostly, books on measurement science just refer to the compensation method very briefly without any theoretical aspects or mathematical formulations at all (Webster, 1999; Dyer, 2001; Klaassen, 2002; Bakshi and Bakshi, 2009; Profos and Pfeifer, 1994; Hoffmann, 2007; Lerch, 2011) or even ignore it (Gosh, 2009; Niebuhr and Lindner, 2002). However, some authors have shown system-based calculations for a specific application. In the work of Krause (Krause, 2004, 2005), a comparison between the deflection and the compensation method specifically applied to a precision balance is accomplished. In Kiencke and Eger (2008), a system-based approach considering selected aspects of the compensation method is outlined briefly. Moreover, the cited references appeared in German and are thus not accessible to an international readership. Hence, a systematic general system-based description with particular respect to important sensor transfer functions, sensor behavior, and sensor properties for practical applications cannot be found in the current literature.

This paper aims at a theoretical description of the compensation method in sensor technology. A system-based approach is used to describe both the deflection and the compensation method. The goal of this study is a general understanding of the system behavior of a closed-loop sensor and to draw specific conclusions for system design and properties toward realization of high sensitivity, suppression of static systematic deviations, fast response, independence of interfering and long-term drift effects like material drift/relaxation and aging. The influence of three different linear standard controller-types, i.e. Proportional (P), Proportional-Integral (PI), and Proportional-IntegralDerivative (PID), on the system behavior is studied. Conclusions are drawn regarding which controller type should be applied for realizing specific sensor properties. The system analysis is carried out in the frequency domain, but is transformed back to the time domain at certain points for better understanding, illustration, and interpretation. At first, a general sensor model is introduced, and different transfer functions, which mainly cover the transfer characteristics of 
current sensors, are proposed. The modified system structure, which results from the feedback for compensation, is shown and the resulting transfer functions are calculated. These transfer functions are systematically analyzed with respect to certain sensor properties mentioned above. The resulting sensor output signal of the compensated sensor is shown in dependence on the controller parameters.

\section{Compensation method in current sensor applications}

In this section, a very brief review of the use of the compensation method in selected current sensor applications shall be given. Mainly, this section is to show that the compensation method can be applied to a broad range of sensor principles. Therefore, selected sensor principles from different fields of application are introduced, and the advantages due to compensation are briefly presented. However, a detailed review or extended description of the respective measurement principles are beyond the scope of this work.

\subsection{Electrodynamic precision balance}

This balance principle is exceedingly widely used and is applied to measurement problems requiring very high precision. The basic working principle is schematically shown in Fig. 2. If a mass $m$ is applied to the tray, the beam deflects. This deflection $x_{\mathrm{c}}$ is detected by an optical position sensor. The coil current $I_{\mathrm{c}}$ generates the force $F_{\mathrm{c}}$ at the lever and is adjusted by the electronic controller in such a way that the deflection $x_{\mathrm{c}}$ in the steady state is zero. Hence, the voltage $V_{\mathrm{M}}$ at the resistor $R_{\mathrm{M}}$ is proportional to the coil current, and thus, to the applied mass $m$. The coil current can be set up far more precisely than the beam deflection can be measured. Therefore, weights can be determined with high precision. Furthermore, the beam is quasi-undeflected during the entire measuring process. A mechanical deficient or indifferent beam is stabilized by the electronic feedback. Consequently, the mechanical properties of the beam will negligibly affect the measurement process (Ştefănescu, 2011).

\subsection{Atomic force microscope in constant force mode}

The atomic force microscope (AFM) ranks among the most versatile methods for the imaging of nanoscale structures in micro/nano electronics or molecular biology, nanomanipulation, and nanoassembly (Bhushan, 2005). Basically, a sharp tip on a flexible cantilever is brought into close proximity to the sample surface, as schematically illustrated in Fig. 3. The resulting interaction between the tip and the sample surface causes a deflection $d$ of the cantilever. This deflection is detected by measuring the reflection of a laser spot off of the backside of the cantilever using a four quadrant photo detector. For an undeflected cantilever, ideally, the spot is detected in the center of the detector. A movement of the

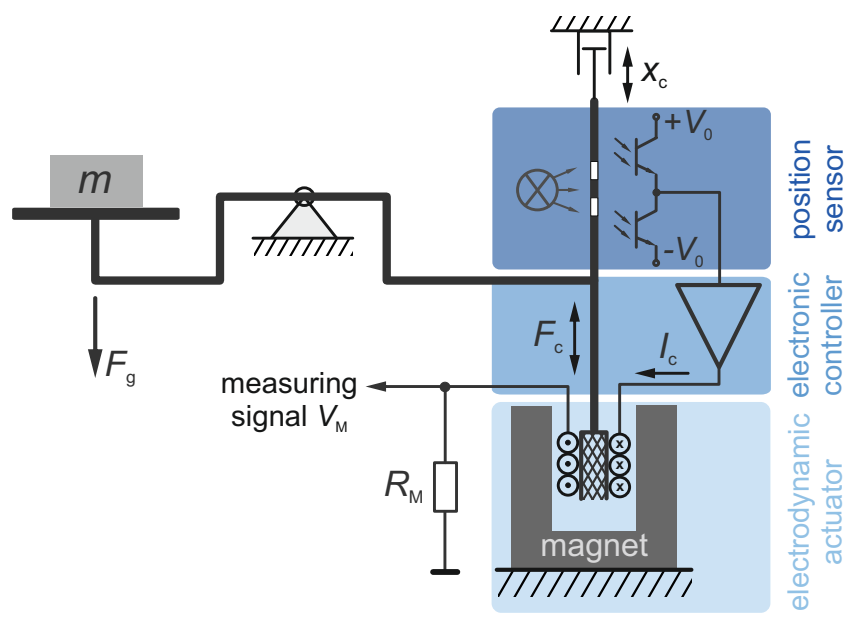

Figure 2. Basic principle of an electrodynamic precision balance according to Krause (2005) and Ştefănescu (2011).

cantilever leads to a shift of the spot position on the detector. Thus, the detector signal is a direct measure of the cantilever deflection. Due to the "optical lever", even small deflections can be detected reliably. In constant force mode, the detector signal is compared to a reference signal and fed to a controller. The provided controller signal, which leads to a $z$-movement of the piezo stage $z_{\mathrm{p}}$, is exactly assessed such that the cantilever deflection is balanced and the force acting on the cantilever remains constant in the steady state. The controller signal is thus a direct estimate of the surface structure. PI- or PID-controllers are widely used in the feedback loop (Abramovitch et al., 2007). However, also other controller types like Proportional-Double-Integral (PII) or Proportional-Double-Integral-Derivative (PIID) controllers have been reported (Abramovitch et al., 2009).

Since the relation between the force acting on the cantilever and the tip-sample-distance is highly non-linear, the closed-loop configuration enables a reliable detection of the sample surface properties. However, the scan speed is limited by the dynamics of the feedback loop.

\subsection{Hot wire anemometer in constant-temperature mode (CTA)}

Hot wire anemometers are indispensable instruments in flow metrology. They are able to measure flow velocity and velocity profiles in liquids and gases. Here, the resistance $R_{\mathrm{w}}$ of a wire probe is changed by the temperature change (heat dissipation $Q$ of the wire) caused by a liquid or gas flow $J$, as illustrated in Fig. 4. Basically, the dissipated thermal energy is a measure of flow velocity. In constant temperature mode, the wire probe is part of a Wheatstone bridge circuit with constant current supply $I_{0}$. Assuming a balanced bridge at a certain condition, a flow of liquid or gas with a certain velocity consequently leads to a heat transfer from the wire probe. This results in a temperature decrease, consequently 


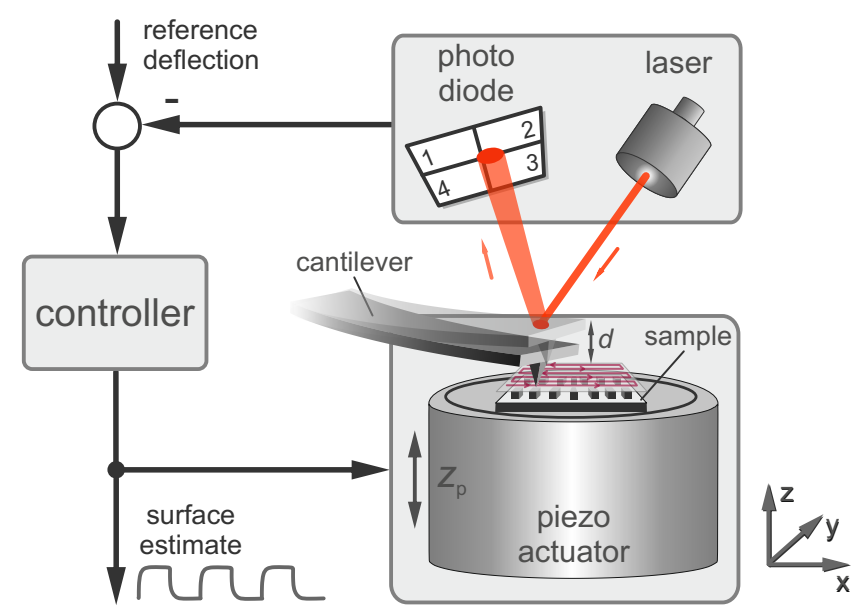

Figure 3. Basic principle of an atomic force microscope in a closedloop configuration (constant force mode).

in an increase in resistivity $\Delta R_{\mathrm{w}}$, and therefore, in an unbalanced state of the bridge circuit $\left(\Delta V_{\mathrm{b}} \neq 0\right)$. The difference voltage $\Delta V_{\mathrm{b}}$ is amplified by a servo amplifier. The resulting output current $I_{\mathrm{A}}$ of the amplifier is fed back to the bridge circuit. This current again induces a temperature increase at the wire probe such that the bridge balance is restored and the wire probe remains at constant temperature at the steady state. In this closed-loop configuration, the amplifier output $I_{\mathrm{A}}$ is a function of the dissipated heat from the sensor wire and indirectly of the flow velocity (Fingerson and Freymuth, 1996).

A high gain servo amplifier enables the measurement of rapid flow velocity fluctuations. The cut-off frequency of an anemometer in constant temperature mode can be about three orders of magnitude higher compared to conventional constant current anemometers (Tavoularis, 2005). Due to the constant wire probe temperature, the effect of the thermal inertia of the wire is greatly minimized compared to an openloop system.

\subsection{Broadband lambda probe}

Lambda probes are widely used for oxygen detection and are mainly employed for the measurement of the air/fuel ratio $\lambda$ in combustion engines in automotive industry, pioneered by Robert Bosch GmbH in the early 1970s. Conventional lambda probes use a galvanic Nernst-cell, composed of a solid electrolyte as oxygen conductor between two platinum electrodes. This cell provides an electrical output voltage $V_{\lambda}$ related to the excess oxygen in the exhaust gas. However, with this configuration only a detection of about $\lambda=1$ is possible. Well above and below this value, the change in the output voltage is only marginal; the transfer characteristics shows a two-step behavior, as shown in Fig. $5 b$.

Broadband lambda probes - as the name suggests - can operate in a broad $\lambda$-range compared to the conventional de-

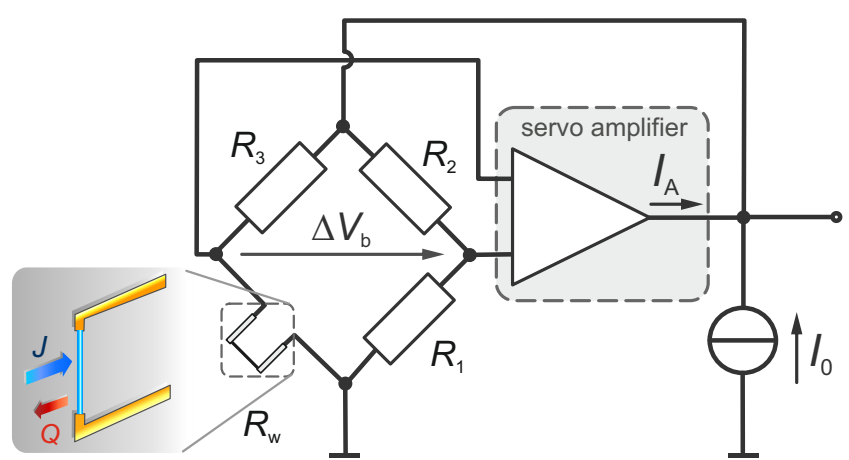

Figure 4. Basic principle of a hot wire anemometer in constant temperature mode (CTA).

sign (Robert Bosch GmbH, 1994). This broadband configuration is schematically shown in Fig. 5a. Here, a sandwich structure comprising a sensing cell, which is identical to the Nernst-cell in the conventional design, a diffusion gap, and a pumping cell are part of a closed-loop control circuit. The exhaust gas is inserted to the diffusion gap via a porous diffusion channel, leading to a certain oxygen content inside the diffusion gap and consequently to a certain $\lambda$-value. The output voltage $V_{\lambda}$ of the sensing cell directly depends on this $\lambda$-value and is compared to the preset reference voltage $V_{\text {ref }}$. The latter is chosen in such a way that it corresponds to $\lambda=1$ $(\approx 450 \mathrm{mV}$ ). Assuming $\lambda>1$ (lean mixture, excess air) in the diffusion gap leads to $V_{\lambda}<V_{\text {ref }}$ and hence to a voltage difference at the input of the amplifier. This voltage difference results in an output current $I_{\mathrm{p}}$. This pumping current pumps excess oxygen ions out of the diffusion gap for compensation to maintain a constant air/fuel ratio of $\lambda=1$. For $\lambda<1$ (rich mixture, lack of air) in the diffusion gap, the pumping current has an opposite sign and causes pumping of oxygen ions into the diffusion gap. Thus, an initially imbalanced oxygen concentration is compensated and the $\lambda$-value inside the diffusion gap remains constant at the steady state. The magnitude and the sign of the pumping current are a measure of $\lambda$.

With this closed-loop configuration, a broad $\lambda$-range $0.7<\lambda<\infty$ can be detected, whereas $\infty$ indicates the oxygen concentration of pure air of $21 \%$ (see Fig. 5c). This enables expanded areas of application apart from its standard use (Bosch GmbH, 2010).

\section{General sensor model with deflection method}

\subsection{Sensor model}

To keep generality, we regard the sensor as a single-input single-output (SISO) system with input $x_{\mathrm{i}}$ and output $x_{\mathrm{o}}$. The transfer behavior is described by the impulse response $g_{\mathrm{s}}$ with $x_{0}(t)=g_{\mathrm{s}} * x_{\mathrm{i}}(t)$, irrespective of any specific sensor principle, as shown in Fig. 6. Here, $x_{\mathrm{i}}$ is the measurand and can be any physical, chemical or biological quantity, whereas $x_{0}$ 


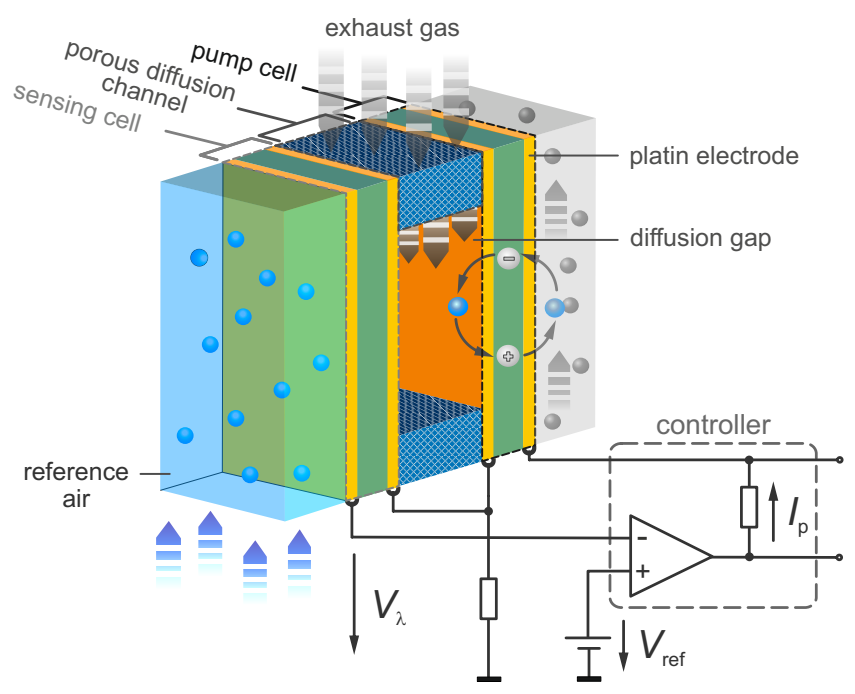

(a)

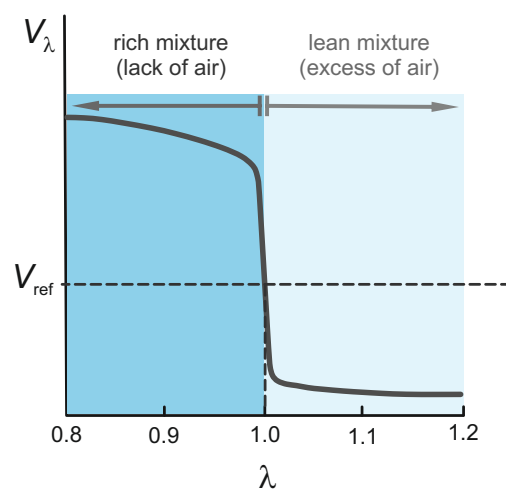

(b)

$$
\begin{gathered}
x_{\mathrm{i}}(t) \rightarrow g_{\mathrm{s}} \rightarrow x_{\mathrm{o}}(t) \\
x_{\mathrm{o}}(t)=g_{\mathrm{s}} * x_{\mathrm{i}}(t)
\end{gathered}
$$

(a)

$$
\begin{gathered}
X_{\mathrm{i}}(s) \longrightarrow \mathrm{G}_{\mathrm{s}} \longrightarrow X_{\mathrm{o}}(s) \\
X_{\mathrm{o}}(s)=G_{\mathrm{s}} \cdot X_{\mathrm{i}}(s)
\end{gathered}
$$

(b)

Figure 6. System model of a sensor; (a) in the time domain, (b) in the frequency domain.

Here, $a_{0}, \ldots, a_{n}$ and $b_{0}, \ldots, b_{m}$ are constant coefficients which are explicitly determined by the sensor properties. However, in consideration of real sensor systems, basically three instrument types, which mainly cover the transfer characteristics of most sensors, can be classified according to their dynamic response as: (I) first-order systems $\left(\mathrm{PT}_{1}\right)$, (II) second-order systems $\left(\mathrm{PT}_{2}\right)$, and (III) differentiating second-order systems ( $\left.\mathrm{PT}_{2}-\mathrm{D}\right)$. Thus, Eq. (1) can be simplified for these three cases. The resulting input-output relations in the time domain are given in Table 2. However, to obtain the output characteristics for a given input signal, the description in the time domain requires the solution of the differential equations. This may result in complicated solution algorithms, especially for more complex systems in closedloop configuration later on. Moreover, the quantitative interpretation of the solution and the extraction of practical conclusions may be not straightforward due to bulky mathematical expressions. Therefore, the sensor transfer characteristics is transformed from the time domain to the frequency domain, which enables a powerful method for theoretical system description (Shinners, 1998). Thus, the sensor can be completely described by its transfer function

$G_{\mathrm{s}}(s)=\frac{X_{\mathrm{o}}(s)}{X_{\mathrm{i}}(s)}=\mathcal{L}\left\{g_{\mathrm{s}}(t)\right\}=\int_{0}^{\infty} g_{\mathrm{s}}(t) e^{-s t} \mathrm{~d} t$.

Figure 5. (a) Schematic illustration of the core section of a broadband lambda probe including the feedback control circuit, according to NGK (2012) (for the sake of simplicity, the heater necessary to heat up the probe to the optimal working temperature is not shown in this illustration); general illustration of the transfer characteristics of a conventional (b) and a broadband lambda probe (c) according to Bosch GmbH (2010).

is assumed to be an electrical output signal (voltage or current) according to the general sensor definition, and $*$ is the convolution operator. Furthermore, we assume the sensor to be a linear time-invariant (LTI) system. The linear dynamic input-output relation of the sensor in the time domain can be completely described by an ordinary linear differential equation of order $n(n \geq m ; n, m \in \mathbb{N})$ with constant coefficients in the following form:

$$
\begin{aligned}
& a_{n} \frac{\mathrm{d}^{n} x_{\mathrm{o}}}{\mathrm{d} t^{n}}+a_{n-1} \frac{\mathrm{d}^{n-1} x_{\mathrm{o}}}{\mathrm{d} t^{n-1}}+\cdots+a_{1} \frac{\mathrm{d} x_{\mathrm{o}}}{\mathrm{d} t}+a_{0} x_{\mathrm{o}}= \\
& b_{m} \frac{\mathrm{d}^{m} x_{\mathrm{i}}}{\mathrm{d} t^{m}}+b_{m-1} \frac{\mathrm{d}^{m-1} x_{\mathrm{i}}}{\mathrm{d} t^{m-1}}+\cdots+b_{1} \frac{\mathrm{d} x_{\mathrm{i}}}{\mathrm{d} t}+b_{0} x_{\mathrm{i}} .
\end{aligned}
$$

Here, $\mathcal{L}$ is the Laplace operator, and $s$ the complex variable. The sensor transfer functions according to Eq. (2) for the three basic sensor types are given in Table 2. The general sensor coefficients in the input-output relations are substituted by the static sensitivity $K$ and the characteristic time constants $T, T_{1}, T_{2}$, and $T_{3}$ of the respective system.

From Table 2 it is apparent that the sensor can be basically described by a quasi-static part and a time-dependent dynamic part as illustrated in the general sensor model in Fig. 7. The sensor model comprises the influence of the measurand $X_{\mathrm{i}}$ (measurement channel) and an interference quantity $Z$ (interference channel). The static sensor behavior can vary from ideal linear behavior $K$ due to the existence of non-linearities, which are apparent in most sensor applications. Both the linear part $K$ and the non-linear part $f$ directly depend on the measurand $X_{\mathrm{i}}$. The static sensitivity is also changed by the interfering quantity $Z$, e.g. temperature in many sensor applications. When no input signal is applied $\left(X_{\mathrm{i}}=0\right)$, only the 
Table 2. Sensor classification and assignment of the respective input-output relations and resulting transfer functions as well as common examples for the respective sensor types.

\begin{tabular}{|c|c|c|c|c|}
\hline & Sensor type & $\begin{array}{l}\text { Input-output relation } \\
x_{\mathrm{o}}(t)=g_{\mathrm{s}} * x_{\mathrm{i}}(t)\end{array}$ & $\begin{array}{l}\text { Sensor transfer function } \\
G_{\mathrm{s}}(s)=X_{\mathrm{o}}(s) / X_{\mathrm{i}}(s)\end{array}$ & Examples \\
\hline $\mathrm{I}:$ & First-order system $\left(\mathrm{PT}_{1}\right)$ & $x_{\mathrm{o}}=\frac{b_{0}}{a_{0}} \cdot \frac{1}{\frac{a_{1}}{a_{0}} \frac{\mathrm{d}}{\mathrm{d} t}+1} \cdot x_{\mathrm{i}}$ & $K \cdot \frac{1}{s T+1}$ & $\begin{array}{l}\text { Temperature sensors } \\
\text { (Fraden, 2010) }\end{array}$ \\
\hline II: & Second-order system $\left(\mathrm{PT}_{2}\right)$ & $x_{\mathrm{o}}=\frac{b_{0}}{a_{0}} \cdot \frac{1}{\frac{a_{2}}{a_{0}} \frac{\mathrm{d}^{2}}{\mathrm{~d} t^{2}}+\frac{a_{1}}{a_{0}} \frac{\mathrm{d}}{\mathrm{d} t}+1} \cdot x_{\mathrm{i}}$ & $K \cdot \frac{1}{T_{1}^{2} s^{2}+T_{2} s+1}$ & $\begin{array}{l}\text { Velocity sensors, } \\
\text { accelerometers } \\
\text { (Fraden, 2010) }\end{array}$ \\
\hline III: & $\begin{array}{l}\text { Differentiating second- } \\
\text { order system }\left(\mathrm{PT}_{2}-\mathrm{D}\right)\end{array}$ & $x_{\mathrm{o}}=a_{0} \cdot \frac{b_{1} \frac{\mathrm{d}}{\mathrm{d} t}}{\frac{a_{2}}{a_{0}} \frac{\mathrm{d}^{2}}{\mathrm{~d} t^{2}}+\frac{a_{1}}{a_{0}} \frac{\mathrm{d}}{\mathrm{d} t}+1} \cdot x_{\mathrm{i}}$ & $K \cdot \frac{s T_{3}}{T_{1}^{2} s^{2}+T_{2} s+1}$ & $\begin{array}{l}\text { Piezoelectric sensors } \\
\text { (Gautschi, 2002), } \\
\text { pyroelectric sensors } \\
\text { (Budzier and Gerlach, 2010) }\end{array}$ \\
\hline
\end{tabular}

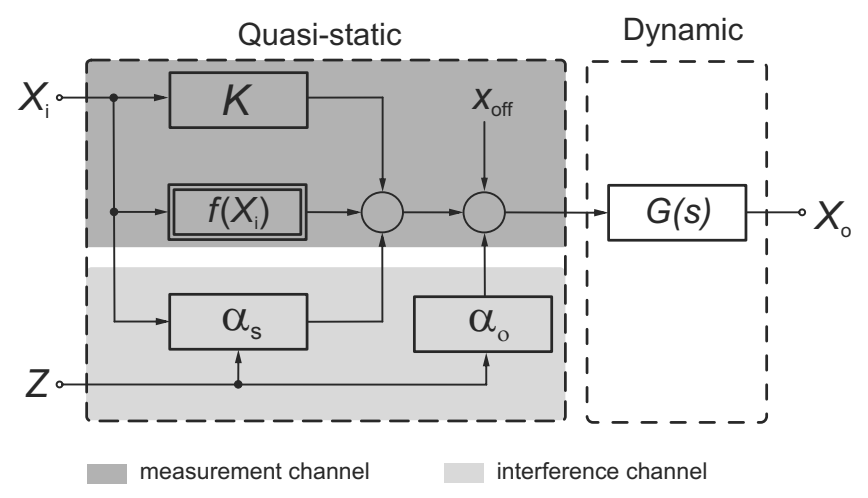

Figure 7. General sensor model with measurand $X_{\mathrm{i}}$ and sensor output $X_{\mathrm{o}}$ sub-divided in a quasi-static and a dynamic part and comprising a measurement and an interference channel. $K$ intrinsic ideal sensitivity, $G(s)$ normalized sensor transfer function, $\alpha_{\mathrm{s}}$ sensitivity coefficient, $Z$ interference quantity altering the ideal sensitivity, $f$ non-linearities in the transfer characteristics, $x_{\text {off }}$ sensor offset, $\alpha_{\mathrm{o}}$ offset coefficient.

static sensor offset $x_{\text {off }}$ is measured as output signal. The sensor offset is independent of the input signal, and thus, does not contain information from the measurand. However, the offset is superimposed by $Z$, and hence, changed by the interference quantity. In most sensors, $x_{\text {off }}$ is primarily altered due to temperature changes. The static sensitivity, the offset and the influence of the interference quantity are additively superimposed, and together with the dynamic sensor part, generating the sensor output signal $X_{0}$. Note that the order of the static and dynamic sensor part is arbitrary and could be also changed. The sensor behavior can be expressed in a general sensor model as

$X_{\mathrm{o}}=\left[(K+\Delta K) \cdot X_{\mathrm{i}}+\left(x_{\mathrm{off}}+\Delta x_{\mathrm{off}}\right)\right] \cdot G(s)$,

with

$\Delta K=f+Z \cdot \alpha_{\mathrm{s}}$ and

$\Delta x_{\mathrm{off}}=Z \cdot \alpha_{\mathrm{o}}$.

It has to be pointed out that the influence of noise is neglected in this model. Based on this sensor model, the effect of the compensation method applied to such a sensor regarding the influence of non-linearities, the interference quantity and static sensitivity changes on the sensor output signal are of interest (see Sect. 4).

\subsection{Quasi-static sensor behavior}

For the description of the quasi-static sensor behavior, we assume that all dynamic processes are terminated and that the system is in an equilibrium state $(G(s)=1$ for $s \rightarrow 0)$. Hence, from Fig. 7 and Eq. (3) it becomes clear that the static sensor output signal is basically determined by two parts. One is the static sensor offset $\tilde{x}_{\text {off }}=\left(x_{\text {off }}+\Delta x_{\text {off }}\right)=\left(x_{\text {off }}+Z \cdot \alpha_{\mathrm{o}}\right)$, which is determined by the intrinsic sensor offset $x_{\text {off }}$ and the interference quantity $Z$, but independent of the input signal $X_{\mathrm{i}}$. The other part is the sensor sensitivity, which is usually altered by non-linearities and the interference quantity $Z$ as a function of the input signal. According to Eq. (3), the resulting sensitivity can be expressed as the superposition of an ideal constant sensitivity $K$ and a deviation $\Delta K$ as $\tilde{K}=(K+\Delta K)$. Here, again two cases have to be considered: (i) a time-independent sensitivity change ( $\Delta K=$ constant) and (ii) a time-dependent sensitivity change (drift) $(\Delta K=\Delta K(s))$. For a time-independent sensitivity change, the interference quantity is constant over time. If one considers small time-independent deviations $\Delta K$ from the ideal behavior, the impact of relative systematic deviations $\Delta K$ on the static sensor output signal can be calculated using a Taylorseries and expressed as a systematic static deviation $\epsilon_{\mathrm{s}}^{\text {defl }}$ as

$\epsilon_{\mathrm{s}}^{\mathrm{defl}}=\frac{\Delta X_{\mathrm{o}}^{\Delta \mathrm{K}, \mathrm{s}}}{X_{\mathrm{o}}}=\frac{1}{X_{\mathrm{o}}} \frac{\partial X_{\mathrm{o}}}{\partial K} \cdot \Delta K=\frac{\Delta K}{K}$ 


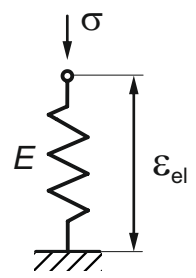

$\varepsilon=\varepsilon_{\mathrm{el}}$

$\frac{\varepsilon(s)}{\sigma(s)}=\frac{1}{E} \triangleq K=$ const.

(a)

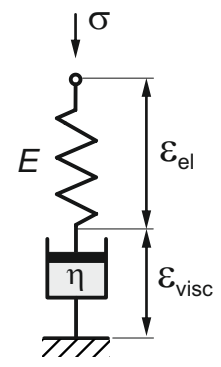

$\varepsilon=\varepsilon_{\mathrm{el}}+\varepsilon_{\mathrm{visc}}$

$\frac{\varepsilon(s)}{\sigma(s)}=\frac{1}{E} \cdot \frac{s+E / \eta}{s} \triangleq K \cdot \frac{s+\delta}{s}$

(b)
Figure 8. Mechanical analog and resulting transfer function of a sensor with (a) ideal linear time-independent sensitivity $K$ (no drift) and (b) time-dependent sensitivity change due to viscoelastic material properties (drift); $\sigma$ mechanical stress, $\epsilon$ mechanical strain, $E$ Young's modulus of the elastic spring, $\eta$ viscosity of the dashpot.

and is equal to the relative sensitivity change.

The second case of a time-dependent sensor sensitivity (drift) is apparent in many sensor applications and results from time-dependent variations of the interference signal. In this paper, this phenomenon is described by a simple model - known from mechanical sensors - which demonstrates the basic idea but keeps simplicity at the same time. The model originates from a simple spring-dashpot arrangement connected in series (Maxwell-model), as illustrated in Fig. 8. This mechanical model represents relaxation effects in spring elements under load due to viscoelastic material properties. The resulting transfer function is used to describe linear drift effects irrespectively of any specific sensor principle. In the frequency domain, $\tilde{K}$ can generally be described as

$\tilde{K}(s)=K \cdot \frac{s+\delta}{s}$,

where $\delta$ is a variable drift rate. Although we consider the static sensor behavior in this section, the phenomenon of drift is a dynamic process occurring in the quasi-static part of the sensor model. Therefore, we can illustrate the timedependent sensitivity by calculating the sensor response due to a step change of the input signal $X_{\mathrm{i}}$ in the time domain, which leads to

$x_{\mathrm{o}}(t)=\mathcal{L}^{-1}\left\{\tilde{K}(s) \cdot \frac{1}{s}\right\}=K(1+\delta t)$.

The normalized step response $x_{\mathrm{o}} / K$ for the model in Eq. (8) for different drift rates $\delta$ is shown in Fig. 9. For a constant input signal, the static sensor output signal should ideally be constant. However, due to the drift phenomenon, the output signal increases with time. Thus, an explicit correlation between the input and the output signal is not possible without

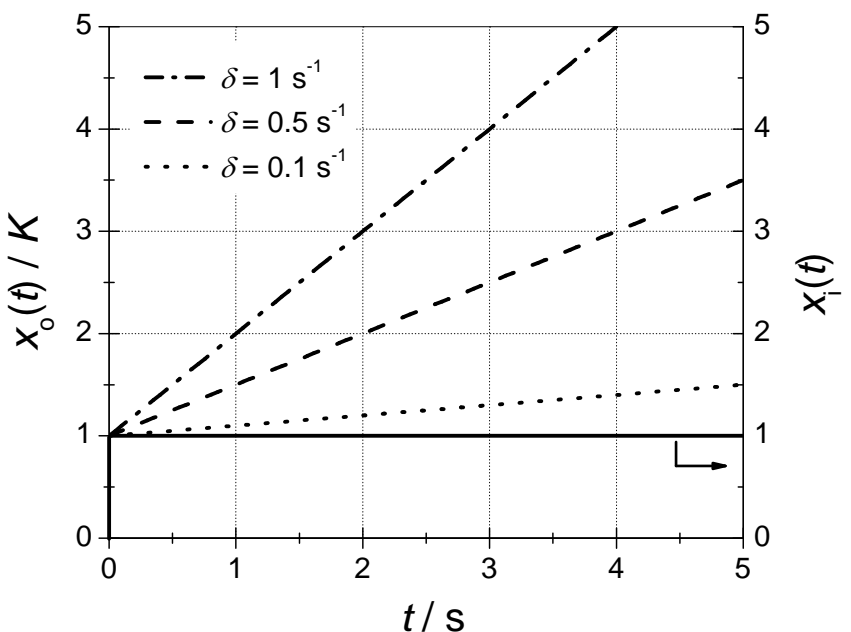

Figure 9. Normalized sensor output signal $x_{\mathrm{o}} / K$ (interrupted lines) for a unit step input (solid line) when considering a time-dependent sensor sensitivity (drift) with different drift rates $\delta$.

additional stipulations, i.e. the knowledge of the drift rate and the establishment of a constant measurement time.

For a time-dependent sensitivity change, the ideal sensitivity $K$ has to be replaced by $\tilde{K}(s)$ from Eq. (7). Thus, the relative deviation of the output signal for a drift-affected sensor according to Eq. (6) becomes

$\epsilon_{\mathrm{d}}^{\mathrm{defl}}=\frac{\Delta X_{\mathrm{o}}^{\Delta \mathrm{K}, \mathrm{d}}}{X_{\mathrm{o}}}=\left(1+\frac{\delta}{S}\right) \frac{\Delta K}{K}$.

For both time-dependent and time-independent sensitivity changes $\Delta K$, it is obvious that the sensor output signal is directly influenced by these deviations. For temporal drift effects, the relative deviation $\epsilon_{\mathrm{d}}^{\text {defl }}(s)$ of the sensor output is time-dependent. For infinite measurement times, the deviation reaches infinity. On the other hand, for $\delta=0$ Eq. (9) becomes Eq. (6).

\subsection{Response time}

Equation (7) describes the time-dependence of the quasistatic sensor parameter $K$ and hence characterizes the unwanted long-term behavior of changing sensor properties, whereas $G(s)$ describes the dynamic short-term behavior. Usually, a response time as short as possible is desired. As quoted in Table 2, sensors can be classified by their transfer function $G(s)$. In general, the dynamic behavior of a sensor depends on the location of the roots of the numerator polynomial (zeros) and the denominator polynomial (poles) in the complex Gauss-plane. In particular, the sensor response time is determined by the dominant pole that is placed closest to the imaginary axis. The poles and zeros are preset by the sensor coefficients $\left(a_{0}, a_{1}, a_{2}, b_{0}, b_{1}\right)$ and consequently by the intrinsic sensor properties. Therefore, the operation of a sensor in deflection mode (open-loop configuration) leads to 


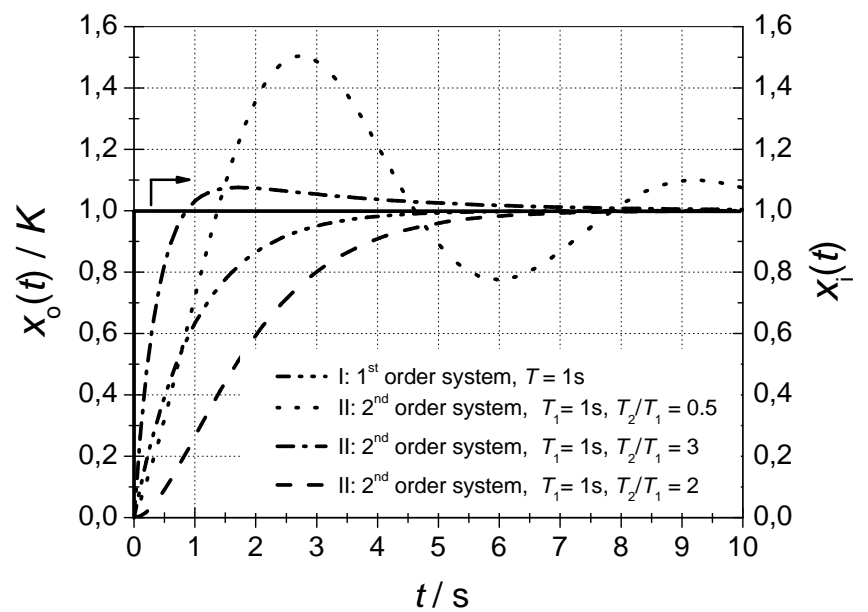

Figure 10. Normalized sensor output signals $x_{\mathrm{o}} / K$ for the different sensor types and different sensor parameters (interrupted lines) for a unit step input (solid line).

a defined dynamic response that cannot be modified. Typical step responses of sensor types I and II with different sensor parameters are exemplarily illustrated in Fig. 10.

\section{General sensor model with compensation method}

\subsection{System model}

Sensors using the compensation method exhibit a closedloop feedback structure where the deflection is brought back to zero by a compensation force. This causes a force equilibrium and keeps the deflection of the sensor - occasionally with a small remnant control deviation - at zero. The deflection can no longer be the measurement signal because it amounts more or less to zero. The sensor signal being proportional to the measurand is now the compensation force or an electrical quantity creating this compensation force. The resulting compensation circuit must at least contain the sensor itself, an amplifier, and the controller/actuator unit. Other components (e.g. D/A converter, filter) may be necessary, but do not alter the general system behavior and are thus neglected in this study. Because we act on the assumption of an electrical sensor output signal, amplification with a highgain amplifier is assumed. For the sake of simplicity, we assume the amplifier, actuator, and controller to be much faster than the sensor itself. This would be particularly the case for, e.g. mechanical and chemical sensors. Hence, we can consider the actuator and the controller as one block placed in the feedback path. For other sensor types, where this simplifying assumption does not hold, the closed-loop sensor model with only one block in the feedback path is nevertheless applicable. In this case, the dominant time constant of the feedback block is a combination of the time constants of the actuator and the controller parameters, respectively. The sensor with compensation is illustrated in Fig. 11. Note

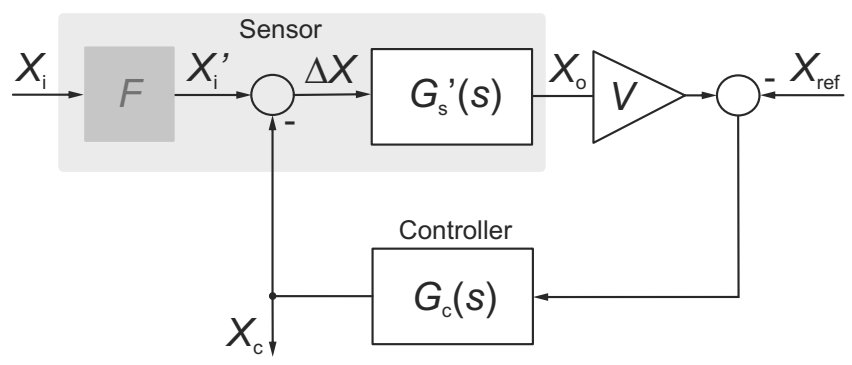

Figure 11. Closed-loop compensation circuit where the sensor output signal $X_{\mathrm{o}}$ is fed back to the sensor input via an amplifier and a controller. $F$ is a transfer element transforming an arbitrary input quantity in a quantity which can be actually compensated.

that the input signal $X_{\mathrm{i}}^{\prime}$ of the feedback structure is not necessarily the measuring quantity $X_{\mathrm{i}}$. If a direct compensation of $X_{\mathrm{i}}$ is not possible, because the desired quantity cannot be generated by an existing device or the generation is too complicated, an equivalent quantity $X_{\mathrm{i}}^{\prime}$, which is correlated to $X_{\mathrm{i}}$ as $X_{\mathrm{i}}^{\prime}=F \cdot X_{\mathrm{i}}$ and which is accessible for compensation must exist. This is a necessary requirement for the applicability of the compensation method to a certain sensor. The correlation between $X_{\mathrm{i}}$ and $X_{\mathrm{i}}^{\prime}$ can be a simple factor (e.g. the gravity of Earth connecting mass and force in a balance) or rather complex (e.g. broadband lambda-probe or closed-loop hydrogelbased sensors (Schulz et al., 2011)), depending on the specific sensor. In any case, the overall system behavior of the compensation circuit is independent of $F$. Therefore, to keep simplicity without loosing generality, we assume $F=1$ and thus, $X_{\mathrm{i}}^{\prime}=X_{\mathrm{i}}$ and $G_{\mathrm{s}}^{\prime}=G_{\mathrm{s}}$ for all further considerations.

Initially, the input signal $X_{\mathrm{i}}$ causes the sensor output signal $X_{\mathrm{o}} . X_{\mathrm{o}}$ is amplified to $V \cdot X_{\mathrm{o}}$, the preset reference value $X_{\text {ref }}$ is subtracted from it and fed back to the controller that generates the compensation quantity $X_{\mathrm{c}}$. Hence, $X_{\mathrm{c}}$ counteracts the sensor input signal. Assuming ideal compensation, the sensor input $X_{\mathrm{i}}-X_{\mathrm{c}}$ is balanced during the whole measuring process in such a way that $\Delta X$ and hence $V \cdot X_{0}-X_{\text {ref becomes zero }}$ at the steady state. Hence, the sensor output $X_{\mathrm{o}}$ remains constant, independent of changes of the input signal. However, the "force" $X_{\mathrm{c}}$, which is necessary to compensate changes of the input signal, depends on the input signal. Therefore, $X_{\mathrm{c}}$ is a measure of the input signal $X_{\mathrm{i}}$ and thus the output signal of the "force"-compensated sensor. The resulting transfer function of the compensation circuit can then be written as

$G_{\mathrm{comp}}(s)=\frac{X_{\mathrm{c}}(s)}{X_{\mathrm{i}}(s)}=\frac{V \cdot G_{\mathrm{s}}(s) \cdot G_{\mathrm{c}}(s)}{1+V \cdot G_{\mathrm{s}}(s) \cdot G_{\mathrm{c}}(s)}=\frac{\tilde{G}(s)}{1+\tilde{G}(s)}$,

with

$\tilde{G}(s)=V \cdot G_{\mathrm{s}}(s) \cdot G_{\mathrm{c}}(s)$

and expressed in a more general form as

$G_{\text {comp }}(s)=\frac{N_{\text {comp }}(s)}{D_{\text {comp }}(s)}=\frac{c_{m} s^{m}+\ldots+c_{1} s+c_{0}}{d_{n} s^{n}+\ldots+d_{1} s+d_{0}}$, 
Table 3. Transfer functions of basic linear standard controller types (Datta et al., 2000).

\begin{tabular}{ll}
\hline Controller type & Transfer function \\
\hline P-controller & $G_{\mathrm{c}}^{\mathrm{P}}(s)=k_{\mathrm{P}}$ \\
PI-controller & $G_{\mathrm{c}}^{\mathrm{PI}}(s)=k_{\mathrm{P}}+\frac{k_{\mathrm{I}}}{s}$ \\
PID-controller & $G_{\mathrm{c}}^{\mathrm{PID}}(s)=k_{\mathrm{P}}+\frac{k_{\mathrm{I}}}{s}+k_{\mathrm{D}} \cdot s$ \\
\hline
\end{tabular}

where both numerator $N_{\text {comp }}$ and denominator $D_{\text {comp }}$ are composed of polynomials. From Eq. (10) one can see that for $G_{\text {comp }} \neq 0$, the compensation quantity $X_{\mathrm{c}}$ can be used as a direct measure of the measuring quantity $X_{\mathrm{i}}$. Because of the feedback loop, a transfer function in the form $\tilde{G} /(1+\tilde{G})$ arises (cp. Table 1), which exibits a completely different structure compared to the simple open-loop transfer function. Moreover, the system properties can now be tuned and adjusted in certain limits via the amplifier gain and the parameters of $G_{\mathrm{c}}$. Thus, $G_{\text {comp }}$ can be regarded as a variably tunable transfer function.

The controller is assumed to be a standard linear P-, PI-, or PID- controller, respectively. In principle, a specific controller could be designed for the task of compensation. However, practically, the predominant majority of all control tasks are realized by standard controllers (Visioli, 2006). Therefore, we focus on those standard controllers. The transfer functions of the three controller types are listed in Table 3. Here, $k_{\mathrm{P}}$ is the $\mathrm{P}$-factor, $k_{\mathrm{I}}$ the I-factor, and $k_{\mathrm{D}}$ the $\mathrm{D}$-factor, with

$k_{\mathrm{I}}=\frac{k_{\mathrm{P}}}{T_{\mathrm{I}}}$ and $k_{\mathrm{D}}=k_{\mathrm{P}} T_{\mathrm{D}}$,

where $T_{\mathrm{I}}$ and $T_{\mathrm{D}}$ are the integral time and the differential time of the controller, respectively. We assume $k_{\mathrm{P}}, k_{\mathrm{I}}$, and $k_{\mathrm{D}}$ to be constant. The application of the controller transfer functions $G_{\mathrm{c}}(s)$ from Table 3 and the sensor transfer functions $G_{\mathrm{s}}(s)$ from Table 2 to Eq. (10) leads to the closed-loop transfer functions of the three sensor types. The resulting transfer functions of the different sensor-controller combinations are listed in Table 4 where $K^{*}=K \cdot V$. In the following, $K^{*} \cdot k_{\mathrm{P}}$ is termed as feedback factor. However, the most important requirement for a technical use of a closedloop system is the system stability, i.e. a bounded input signal leads to a bounded output signal (BIBO-stability) (Shinners, 1998). Stability has to be guaranteed for all operating conditions. This necessitates the analysis of the closed-loop transfer functions to determine the parameter range in which the system is stable.

\subsection{Stability requirements}

Stability analysis is easily accessible in the frequency domain by analysis of the closed-loop transfer function $G_{\text {comp }}(s)$, as stated in Eq. (12). This transfer function is asymptotically stable if and only if

- $G_{\text {comp }}$ is proper, i.e. the degree $m$ of the numerator $N_{\text {comp }}$ is smaller or equal to the degree $n$ of the denominator $D_{\text {comp }}(m \leq n$, see Eq. 12), and

- if all poles of $G_{\text {comp }}$ are placed in the complex left open half-plane (LOH).

The first stability condition can be easily evaluated by a simple look at the respective closed-loop transfer function (Table 4). The second stability condition can be verified by a stability criterion (e.g. Routh criterion (Shinners, 1998) as used within this work) applied to the denominator polynomial $\mathrm{D}_{\text {comp }}(s)$. Here, the necessary condition is that all coefficients $d_{v}$ of the denominator have the same sign (Stodola condition: Stodola, 1894; Hurwitz, 1895) (e.g. $d_{m}, \ldots, d_{0}>0$ ). The sufficient condition results from the employed stability criterion and has to be evaluated subsequently. If the numerator degree amounts to $n \leq 2$, the Stodola condition is also sufficient. For that simplified case, no stability criterion has to be used in addition.

Beside the fundamental necessity for system stability, we additionally claim a minimum-phase system. Thus, all zeros of $G_{\text {comp }}$ have to be located in the complex left half-plane, including the imaginary axis. In this case, the same criterion (second criterion above) as for the poles is valid, except that the coefficients $c_{v}$ of the numerator can be equal to zero as well $\left(c_{m}, \ldots, c_{0} \geq 0\right)$. A closed-loop transfer function exhibiting nonminimum-phase zeros (zeros in the open right half plane) will show initial overshoot or undershoot, i.e. the step response initially shows an inverse response, spending part of its time going in the "wrong" direction until it changes its direction towards the steady state. This kind of step response exhibits nonmonotonic behavior. This inverse response exists if the transfer function has at least one zero in the right half-plane. Depending on the number of zeros in the right half-plane, the inverse response can become quite complex and can exhibit more direction reversals and zerocrossings (time-dependent signal passes through the value of zero) (Hoagg and Bernstein, 2007; Stewart and Davison, 2006). This behavior is inadequate for sensor applications because the steady state should be reached as fast as possible and a monotone step response is aspired. Therefore, the controller parameters have to be chosen in such a manner that the asymptotically stable closed-loop transfer function shows minimum-phase behavior at the same time. From Table 4 it is apparent that the first stability criterion is fulfilled for all transfer functions. For real sensors, $K>0, V>0$, and $T>0$ can be assumed without limitation. Using these assumptions, first the range of the control parameters which guarantee BIBO-stability is calculated by utilization of the second stability criterion. Subsequently, the parameter range to assure a minimum-phase behavior is calculated. The calculated results are separately listed in Table 5, and finally the resulting 
Table 4. Closed-loop transfer functions $G_{\text {comp }}(s)$ for all sensor-controller combinations considered; $K^{*}=K \cdot V$.

\begin{tabular}{lccc}
\hline$G_{\text {comp }}(s)$ & $\mathrm{P}$ & $\mathrm{PI}$ & $\mathrm{PID}$ \\
\hline $\mathrm{I}: \mathrm{PT}_{1}$ & $\frac{K^{*} k_{\mathrm{P}}}{s T+\left(K^{*} k_{\mathrm{P}}+1\right)}$ & $\frac{K^{*}\left(k_{\mathrm{p}} s+k_{\mathrm{I}}\right)}{s^{2} T+\left(1+K^{*} k_{\mathrm{P}}\right) s+K^{*} k_{\mathrm{I}}}$ & $\frac{K^{*}\left(k_{\mathrm{D}} s^{2}+k_{\mathrm{p}} s+k_{\mathrm{I}}\right)}{\left(T+K^{*} k_{\mathrm{D}}\right) s^{2}+\left(1+K^{*} k_{\mathrm{P}}\right) s+K^{*} k_{\mathrm{I}}}$ \\
II: $\mathrm{PT}_{2}$ & $\frac{K^{*} k_{\mathrm{P}}}{T_{1}^{2} s^{2}+T_{2} s+\left(1+K^{*} k_{\mathrm{P}}\right)}$ & $\frac{K^{*}\left(k_{\mathrm{p}} s+k_{\mathrm{I}}\right)}{T_{1}^{2} s^{3}+T_{2} s^{2}+\left(1+K^{*} k_{\mathrm{P}}\right) s+K^{*} k_{\mathrm{I}}}$ & $\frac{K^{*}\left(k_{\mathrm{D}} s^{2}+k_{\mathrm{P}} s+k_{\mathrm{I}}\right)}{T_{1}^{2} s^{3}+\left(T_{2}+K^{*} k_{\mathrm{D}}\right) s^{2}+\left(1+K^{*} k_{\mathrm{P}}\right) s+K^{*} k_{\mathrm{I}}}$ \\
III: $\mathrm{PT}_{2}$-D & $\frac{K^{*} k_{\mathrm{P}} T_{3} s}{T_{1}^{2} s^{2}+\left(T_{2}+K^{*} k_{\mathrm{P}} T_{3}\right) s+1}$ & $\frac{K^{*} T_{3}\left(k_{\mathrm{p}} s+k_{\mathrm{I}}\right)}{T_{1}^{2} s^{2}+\left(T_{2}+K^{*} k_{\mathrm{P}} T_{3}\right) s+\left(1+K^{*} k_{\mathrm{I}} T_{3}\right)}$ & $\frac{K^{*} T_{3}\left(k_{\mathrm{D}} s^{2}+k_{\mathrm{P}} s+k_{\mathrm{I}}\right)}{\left(T_{1}^{2}+K^{*} k_{\mathrm{D}} T_{3}\right) s^{2}+\left(T_{2}+K^{*} k_{\mathrm{P}} T_{3}\right) s+\left(1+K^{*} k_{\mathrm{I}} T_{3}\right)}$ \\
\hline
\end{tabular}

parameter range meeting both requirements is stated. As can be seen in Table 5, for the combination of a sensor with $\mathrm{PT}_{2}$ behavior together with a PI- or PID-controller, respectively, the degree of the denominator is of order three. Because of that, the Routh criterion derived from the Routh table has been used to get the sufficient stability condition. Stability analysis by utilization of the Routh criterion is explained in more detail in Appendix A. From these considerations it turns out that the range of controller parameters guaranteeing stability depends on the sensor properties. Note that the second stability condition is even satisfied if certain parameters exhibit negative values. However, if one claims stability and minimum-phase behavior at the same time, the controller parameters do not depend on the sensor properties. The parameters can be any positive value or even equal zero, except for the combination of a $\mathrm{PT}_{2}$ element with a PI- or a PIDcontroller. This enables a wide range of possible controller parameters for the adjustment of the desired system behavior for specific applications. Moreover, these findings do not show any contradiction towards the application of the compensation method in a technical measurement system. However, from a practical point of view, the static feedback gain cannot be increased ad infinitum. Above a certain critical gain, a finite lag of the feedback path will lead to instabilities.

\subsection{Steady state behavior}

\subsubsection{Static deviation from ideal compensation}

The designated aim of the compensation method is ideal compensation of the sensor input $X_{\mathrm{i}}$, such that $V \cdot X_{\mathrm{o}}-X_{\text {ref }}$ becomes zero at the steady state $(s \rightarrow 0, t \rightarrow \infty)$. For the sake of simplicity, let us assume $X_{\text {ref }}=0$. Then,

$\Delta X=X_{\mathrm{i}}-X_{\mathrm{c}}=X_{\mathrm{i}}-\left(V \cdot G_{\mathrm{s}} \cdot G_{\mathrm{c}} \cdot \Delta X\right)$

should be consequently as small as possible. From Eq. (14) we can set up a transfer function $G^{\Delta}$ between the compensation deviation $\Delta X$ and the input signal $X_{\mathrm{i}}$ as

$G^{\Delta}:=G_{X_{\mathrm{i}}}^{\Delta X}=\frac{\Delta X(s)}{X_{\mathrm{i}}(s)}=\frac{1}{1+V \cdot G_{\mathrm{s}} \cdot G_{\mathrm{c}}}$.

Since Eq. (15) comprises the same denominator as the general transfer function in Eq. (10), we can act on the assumption that all poles of $G^{\Delta}$ exhibit negative real part (for the parameter range in Table 5), and that the transfer function is thus stable. In this case, one can apply the final value theorem of the Laplace transform, generally written as

$\lim _{t \rightarrow \infty} x(t)=\lim _{s \rightarrow 0}[s \cdot X(s)]$

Using Eq. (16), we can calculate the deviation $\Delta x(t \rightarrow \infty)$ from ideal compensation in the steady state for a step change of $X_{\mathrm{i}}$ as

$\Delta x(\infty)=\lim _{t \rightarrow \infty} \Delta x(t)=\lim _{s \rightarrow 0}\left[s \cdot G^{\Delta}(s) \cdot \frac{1}{s}\right]=\lim _{s \rightarrow 0} G^{\Delta}(s)$.

Example: $\mathrm{PT}_{1}$-type sensor with $\mathrm{P}$-controller

For the simplest example of a $\mathrm{PT}_{1}$-type sensor in combination with a P-controller, $G^{\Delta}$ is

$G^{\Delta}(s)=\frac{s T+1}{s T+K^{*} k_{\mathrm{P}}+1}$,

leading to

$\Delta x(\infty)=\lim _{s \rightarrow 0}\left(\frac{s T+1}{s T+K^{*} k_{\mathrm{P}}+1}\right)=\frac{1}{1+K^{*} k_{\mathrm{P}}}$.

The static deviation from ideal compensation for all sensorcontroller combinations are given in Table 6. From Table 6 one can see the same behavior for both $\mathrm{PT}_{1}$ - and $\mathrm{PT}_{2}$-type sensors regarding the static deviation from ideal compensation. For application of a P-controller, $\Delta x$ depends on the feedback factor. For unity feedback $\left(K^{*} k_{\mathrm{P}}=1\right)$, a deviation of $50 \%$ is observed. However, for an increasing feedback factor, the deviation decreases and converges to zero for a highgain feedback. A deviation of only $1 \%$ is already reached with a feedback factor of 100 . For the application of a PIor PID-controller, $\Delta x$ turns out to be zero, and thus, independent of the feedback parameters. Therefore, a PI- or PIDcontroller should be used for these sensor types in order to achieve full compensation. The combination of a $\mathrm{PT}_{2}$-D-type sensor with a P-controller cannot be used for sensor applications. This can be easily understood by application of the final value theorem to the respective transfer function in Table 4 . In this case, the sensor output at the steady state always equals zero independent of the input signal. Thus, different input signals would always lead to the same (zero) output 
Table 5. Parameter range guaranteeing BIBO-stability and a minimum-phase system, and the resulting combined parameter range satisfying both requirements for all considered sensor-controller combinations.

\begin{tabular}{|c|c|c|c|}
\hline$G_{\text {comp }}(s)$ & $\mathrm{P}$ & PI & PID \\
\hline \multicolumn{4}{|c|}{ Parameter range for a BIBO-stable system } \\
\hline $\mathrm{I}: \mathrm{PT}_{1}$ & $k_{\mathrm{P}}>-\frac{1}{K^{*}}$ & $k_{\mathrm{P}}>-\frac{1}{K^{*}}, k_{\mathrm{I}}>0$ & $k_{\mathrm{P}}>-\frac{1}{K^{*}}, k_{\mathrm{I}}>0, k_{\mathrm{D}}>-\frac{T}{K^{*}}$ \\
\hline \multirow[t]{2}{*}{ II: $\mathrm{PT}_{2}$} & $k_{\mathrm{P}}>-\frac{1}{K^{*}}$ & $k_{\mathrm{P}}>-\frac{1}{K^{*}}, k_{\mathrm{I}}>0$ & $k_{\mathrm{P}}>-\frac{1}{K^{*}}, k_{\mathrm{I}}>0, k_{\mathrm{D}}>-\frac{T_{2}}{K^{*}}$ \\
\hline & & $1+K^{*} k_{\mathrm{P}}-\frac{T_{1}^{2} K^{*} k_{1}}{T_{2}}>0^{\mathrm{a}}$ & $1+K^{*} k_{\mathrm{P}}-\frac{T_{1}^{2} K^{*} k_{\mathrm{I}}}{T_{2}+K^{*} k_{\mathrm{D}}}>0^{\mathrm{a}}$ \\
\hline III: $\mathrm{PT}_{2}$-D & $k_{\mathrm{P}}>-\frac{T_{2}}{K^{*} T_{3}}$ & $k_{\mathrm{P}}>-\frac{T_{2}}{K^{*} T_{3}}, k_{\mathrm{I}}>-\frac{1}{K^{*} T_{3}}$ & $k_{\mathrm{P}}>-\frac{T_{2}}{K^{*} T_{3}}, k_{\mathrm{I}}>-\frac{1}{K^{*} T_{3}}, k_{\mathrm{D}}>-\frac{T_{1}^{2}}{K^{*} T_{3}}$ \\
\hline \multicolumn{4}{|c|}{ Parameter range for a minimum-phase system } \\
\hline I-III: & $k_{\mathrm{P}} \geq 0$ & $k_{\mathrm{P}} \geq 0, k_{\mathrm{I}} \geq 0$ & $k_{\mathrm{P}} \geq 0^{\mathrm{b}}, k_{\mathrm{I}} \geq 0, k_{\mathrm{D}} \geq 0$ \\
\hline \multicolumn{4}{|c|}{ Resulting combined parameter range } \\
\hline $\mathrm{I}: \mathrm{PT}_{1}$ & $k_{\mathrm{P}}>0^{\mathrm{c}}$ & $k_{\mathrm{P}} \geq 0, k_{\mathrm{I}}>0$ & $k_{\mathrm{P}}>0^{\mathrm{d}}, k_{\mathrm{I}}>0, k_{\mathrm{D}} \geq 0$ \\
\hline \multirow[t]{2}{*}{ II: $\mathrm{PT}_{2}$} & $k_{\mathrm{P}}>0$ & $k_{\mathrm{P}} \geq 0, k_{\mathrm{I}}>0$ & $k_{\mathrm{P}}>0, k_{\mathrm{I}}>0, k_{\mathrm{D}} \geq 0$ \\
\hline & & $T_{2}\left(1+K^{*} k_{\mathrm{P}}\right)>T_{1}^{2} K^{*} k_{\mathrm{I}}{ }^{\mathrm{a}}$ & $\left(T_{2}+K^{*} k_{\mathrm{D}}\right)\left(1+K^{*} k_{\mathrm{P}}\right)>T_{1}^{2} K^{*} k_{\mathrm{I}}^{\mathrm{a}}$ \\
\hline III: $\mathrm{PT}_{2}-\mathrm{D}$ & $k_{\mathrm{P}}>0$ & $k_{\mathrm{P}} \geq 0, k_{\mathrm{I}} \geq 0$ & $k_{\mathrm{P}}>0^{\mathrm{d}}, k_{\mathrm{I}} \geq 0, k_{\mathrm{D}} \geq 0$ \\
\hline
\end{tabular}

Table 6. Static deviation from ideal compensation for all considered sensor-controller combinations.

\begin{tabular}{lccc}
\hline$\Delta x(\infty)$ & $\mathrm{P}$ & $\mathrm{PI}$ & $\mathrm{PID}$ \\
\hline $\mathrm{PT}_{1}$ & $\frac{1}{1+K^{*} k_{\mathrm{P}}}$ & 0 & 0 \\
$\mathrm{PT}_{2}$ & $\frac{1}{1+K^{*} k_{\mathrm{P}}}$ & 0 & 0 \\
$\mathrm{PT}_{2}-\mathrm{D}$ & N.A. & $\frac{1}{K^{*} T_{2} k_{\mathrm{I}}+1}$ & $\frac{1}{K^{*} T_{2} k_{\mathrm{I}}+1}$ \\
\hline
\end{tabular}

signal. However, a PI- or a PID-controller can be successfully applied. The application of PI- or PID-controller to this sensor type leads to a static deviation that depends on the feedback factor, the integral time $T_{\mathrm{I}}$ and the time constant $T_{2}$. Thus, for a high feedback factor and a small integral time, the static deviation converges to zero.

\subsubsection{Influence of static parameter variations}

For a static analysis of the compensation circuit, we assume the closed-loop system to be in a steady state $\left(G_{\text {comp }}^{\text {stat }}=\right.$ $\left.G_{\text {comp }}(s \rightarrow 0)\right)$. So far, we have considered an ideal sensitivity $K$ for the transfer function of the compensation circuit. However, as described in the general sensor model in Sect. 3.1, the sensitivity is usually influenced by the interference quantity. Furthermore, compared to the open-loop sensor, the am- plifier and the controller parameters can exhibit deviations from ideal behavior as well. Considering small static variations, the impact of these deviations on the output signal $X_{\mathrm{c}}$ can be calculated by a first-order Taylor-series as

$\epsilon_{\mathrm{s}}^{\mathrm{comp}}=\frac{\Delta X_{\mathrm{c}}}{X_{\mathrm{c}}}=\frac{1}{X_{\mathrm{c}}}\left(\frac{\partial X_{\mathrm{c}}}{\partial K} \cdot \Delta K+\frac{\partial X_{\mathrm{c}}}{\partial V} \cdot \Delta V+\sum_{i=1}^{3} \frac{\partial X_{\mathrm{c}}}{\partial k_{i}} \cdot \Delta k_{i}\right)$.

Here, $k_{i} \in\left\{k_{\mathrm{P}}, k_{\mathrm{I}}, k_{\mathrm{D}}\right\}$ and $\Delta k_{i} \in\left\{\Delta k_{\mathrm{P}}, \Delta k_{\mathrm{I}}, \Delta k_{\mathrm{D}}\right\}$ are the controller parameters and their systematic deviations, respectively, depending on which controller type is used.

\section{Example: $\mathrm{PT}_{1}$-type sensor with $\mathrm{P}$-controller}

For the simplest example of a $\mathrm{PT}_{1}$-type sensor in combination with a P-controller, $\epsilon_{\mathrm{s}}^{\text {comp }}$ is

$$
\begin{aligned}
\epsilon_{\mathrm{s}}^{\text {comp }}= & \frac{1+K V k_{\mathrm{P}}}{K V k_{\mathrm{P}}}\left[\frac{V k_{\mathrm{P}}}{\left(1+K V k_{\mathrm{P}}\right)^{2}} \Delta K+\frac{K k_{\mathrm{P}}}{\left(1+K V k_{\mathrm{P}}\right)^{2}} \Delta V\right. \\
& \left.+\frac{V K}{\left(1+K V k_{\mathrm{P}}\right)^{2}} \Delta k_{\mathrm{P}}\right] \\
= & \frac{1}{1+K^{*} k_{\mathrm{P}}}\left(\frac{\Delta K}{K}+\frac{\Delta V}{V}+\frac{\Delta k_{\mathrm{P}}}{k_{\mathrm{P}}}\right) .
\end{aligned}
$$

To allow an easy comparison with the open-loop sensor, the deviations which do not result from the sensor itself can be 


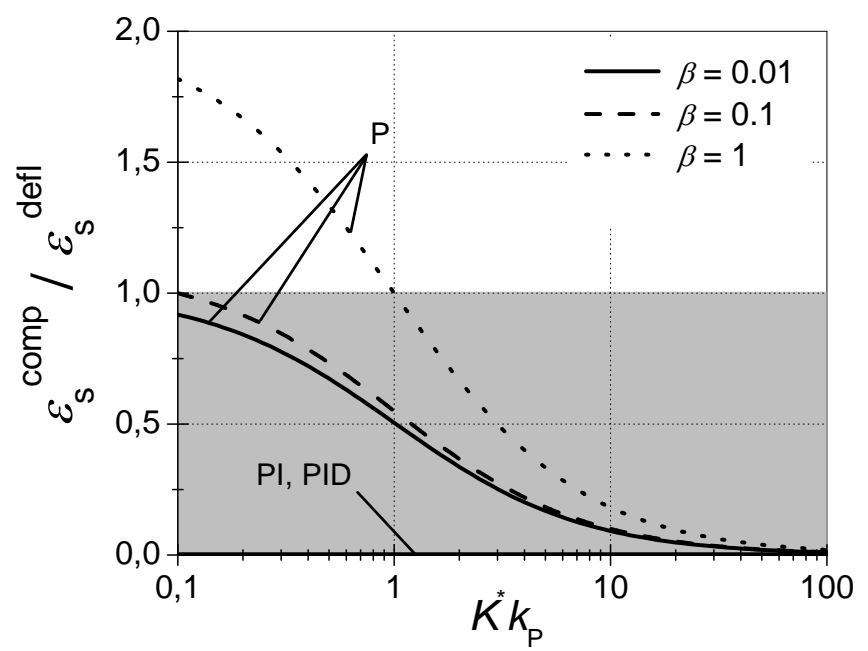

Figure 12. Ratio between systematic static deviations of the closedloop sensor and the open-loop sensor versus the feedback factor $K^{*} k_{\mathrm{P}}$ for a $\mathrm{PT}_{1}$-type sensor in combination with all three controller types. $\beta$ indicates different deviation contributions from the feedback elements compared to the deviation of the sensor itself.

summarized as a feedback deviation $\triangle F B$ and can generally be described as a multiple $(\beta)$ of the sensor deviation as

$\frac{\Delta F B}{F B}=\frac{\Delta V}{V}+\frac{\Delta k_{\mathrm{P}}}{k_{\mathrm{P}}}=\beta \cdot \frac{\Delta K}{K}$.

Thus, Eq. (21) becomes

$\epsilon_{\mathrm{s}}^{\mathrm{comp}}=\frac{1+\beta}{1+K^{*} k_{\mathrm{P}}} \frac{\Delta K}{K}$.

The ratio between the relative systematic static deviation of the closed-loop compensation circuit and the open-loop sensor is

$\frac{\epsilon_{\mathrm{s}}^{\text {comp }}}{\epsilon_{\mathrm{S}}^{\mathrm{defl}}}=\frac{1+\beta}{1+K^{*} k_{\mathrm{P}}}$.

This ratio is illustrated in Fig. 12 as a function of the feedback factor, and of $\beta$, indicating the deviation contribution of the feedback elements. Usually, the feedback elements should be designed such that systematic deviations are much smaller than the deviations of the sensor itself. As can be seen from Fig. 12, if the feedback deviations are just $1 \%$ $(\beta=0.01)$ of the sensor deviations, a unity feedback already minimizes the overall deviation to $50 \%$ compared to an open-loop sensor. However, if the circuit comprises higher feedback deviations, the feedback factor can be systematically increased to achieve a specified lower deviation of the output signal. If one wants to achieve a constant uncertainty, the required feedback factor linearly increases with $\beta$, as stated in Eq. (24).

The ratios between the deviations of the closed-loop configuration compared to the open-loop sensor for all sensorcontroller combinations are listed in Table 7. From this table,
Table 7. Systematic static deviations of the closed-loop sensor for all sensor-controller combinations.

\begin{tabular}{lccc}
\hline$\epsilon_{\mathrm{s}}^{\text {comp }}$ & $\mathrm{P}$ & $\mathrm{PI}$ & $\mathrm{PID}$ \\
\hline $\mathrm{PT}_{1}$ & $\frac{\frac{\Delta K}{K}+\frac{\Delta V}{V}+\frac{\Delta k_{\mathrm{P}}}{k_{\mathrm{p}}}}{1+K^{*} k_{\mathrm{P}}}$ & 0 & 0 \\
$\mathrm{PT}_{2}$ & $\frac{\frac{\Delta K}{K}+\frac{\Delta V}{V}+\frac{\Delta k_{\mathrm{P}}}{k_{\mathrm{P}}}}{1+K^{*} k_{\mathrm{P}}}$ & 0 & 0 \\
$\mathrm{PT}_{2}-\mathrm{D}$ & N.A. & $\frac{\frac{\Delta K}{K}+\frac{\Delta V}{V}+\frac{\Delta k_{\mathrm{I}}}{k_{\mathrm{I}}}}{1+K^{*} k_{\mathrm{P}} \frac{T_{3}}{T_{\mathrm{I}}}}$ & $\frac{\frac{\Delta K}{K}+\frac{\Delta V}{V}+\frac{\Delta k_{\mathrm{I}}}{k_{\mathrm{I}}}}{1+K^{*} k_{\mathrm{P}} \frac{T_{3}}{T_{\mathrm{I}}}}$ \\
\hline
\end{tabular}

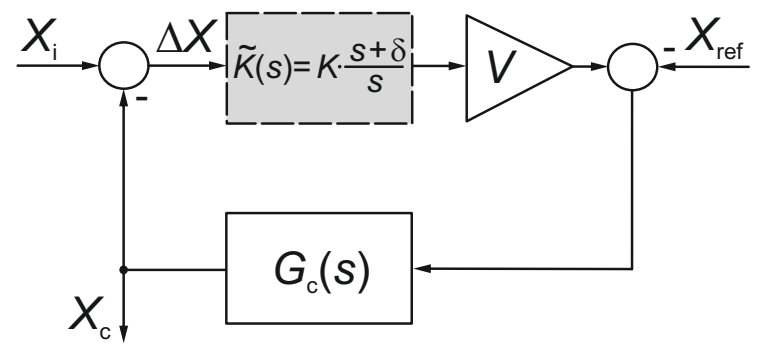

Figure 13. Closed-loop compensation circuit where the quasi-static sensor part is characterized by a sensitivity $\tilde{K}$, describing the dynamics of the drift process by a drift model.

one can see that for $\mathrm{PT}_{1}$ - and $\mathrm{PT}_{2}$-type sensors in combination with a P-controller, the systematic deviations depend on the deviation contributions of the single circuit elements and the feedback factor. However, by additional application of an I-part (PI- or PID-controller), the systematic deviations can be completely eliminated and no longer depend on the deviations of the circuit elements. For a $\mathrm{PT}_{2}$-D-sensor in combination with a PI- or PID-controller, a similar behavior as discussed for the P-controller before can be observed. Here, the overall deviation of the output signal cannot only be tuned by the static amplification $V$ and the P-factor $k_{\mathrm{P}}$, but additionally by the integral time $T_{\mathrm{I}}$. The smaller the integral time is, the better is the resulting suppression of deviations.

\subsubsection{Influence of time-dependent parameter variations}

If the external interference quantity varies with time, the long-term sensor output signal shows a continuous drift even if the dynamic sensor part is already equilibrated. The dynamics of the drift process is modeled by the transfer function $\tilde{K}(s)$. The application of the drift model from Eq. (7) to the closed-loop compensation circuit is illustrated in Fig. 13. The closed-loop transfer functions according to Eq. (10), considering all three controller types, are calculated and listed in Table 8 . From Table 8 one can observe that the unstable transfer function of the open-loop sensor exhibiting drift in Eq. (7) is transformed into a more complex transfer function. Here, the coefficients of the numerator and the denominator can be tuned. We also find that all three closed-loop 
Table 8. Closed-loop transfer function $G_{\text {comp }}^{\Delta \mathrm{d}, \mathrm{d}}$ considering a timedependent sensitivity $\tilde{K}$ instead of an ideal static sensitivity $K$ by application of the drift model from Eq. (7) to the static sensor part (grey box in Fig. 13).

\begin{tabular}{ll}
\hline & $G_{\mathrm{comp}}^{\Delta \mathrm{K}, \mathrm{d}}(s)$ \\
\hline $\mathrm{P}$ & $\frac{K^{*} k_{\mathrm{P}}(s+\delta)}{\left(1+K^{*} k_{\mathrm{p}}\right) s+K^{*} k_{\mathrm{p}} \delta}$ \\
PI & $\frac{K^{*} k_{\mathrm{p}} s^{2}+\left(K^{*} k_{\mathrm{I}}+K^{*} k_{\mathrm{p}} \delta\right) s+K^{*} k_{1} \delta}{\left(1+K^{*} k_{\mathrm{P}}\right) s^{2}+\left(K^{*} k_{\mathrm{I}}+K^{*} k_{\mathrm{P}} \delta\right) s+K^{*} k_{\mathrm{I}} \delta}$ \\
PID & $\frac{K^{*} k_{\mathrm{D}} s^{3}+\left(K^{*} k_{\mathrm{p}}+K^{*} k_{\mathrm{D}} \delta\right) s^{2}+\left(K^{*} k_{\mathrm{I}}+K^{*} k_{\mathrm{p}} \delta\right) s+K^{*} k_{\mathrm{I}} \delta}{K^{*} k_{\mathrm{D}} s^{3}+\left(1+K^{*} k_{\mathrm{P}}+K^{*} k_{\mathrm{D}} \delta\right) s^{2}+\left(K^{*} k_{\mathrm{I}}+K^{*} k_{\mathrm{P}} \delta\right) s+K^{*} k_{\mathrm{I}} \delta}$ \\
\hline
\end{tabular}

transfer functions are exactly proper. The parameter range enabling a stable as well as a minimum-phase system is determined according to the criteria in Sect. 4.2 and listed in Table B1. From this table one can see that the system can be operated in a broad parameter range without jeopardizing the system stability. For the utilization of a P- or PI-controller, the controller parameters have to be simply positive to assure a stable minimum-phase system. For a PID-controller, the restrictions seem more complicated. However, if one simplifies the expression arising from the Routh table, one finds that again all of the stated requirements are met for positive controller parameters $\left(k_{\mathrm{P}}, k_{\mathrm{I}}, k_{\mathrm{D}}>0\right)$ independent of the drift-rate (see Sect. B1 in Appendix B). In this sense, stability directly involves a system without drift, because a system exhibiting drift is a priori unstable. This can be shown by calculating the steady state behavior of the output signal $\left(x_{\mathrm{c}}\right)$ considering a unit step input according to Eq. (16) as

$x_{\mathrm{c}}(\infty)=\lim _{t \rightarrow \infty} x_{\mathrm{c}}(t)=\lim _{s \rightarrow 0} G_{\mathrm{comp}}^{\Delta \mathrm{K}}(s)=1$

and is valid for all three controller types. One can see that the output signal of the closed-loop system is constant at the steady state for all three controller types, whereas the output signal of the open-loop sensor diverges to infinity (cp. Fig. 9).

To enable a quantitative comparison of the quality of drift suppression for the three different controller types in dependence on the controller parameters, the use of a global performance index is reasonable. A performance index, which is easy to handle and meaningful at the same time, is the Integral Square Error (ISE) (Newton et al., 1964) which can be written in the time domain as

$J=\int_{0}^{\infty} \Delta x^{2}(t) \mathrm{d} t$.

Here, $\Delta x(t)$ is the difference between the input step $x_{\mathrm{i}}=$ $1(t)$ and the actual sensor output signal $x_{\mathrm{c}}$ (cp. Fig. 13). This integral is a function of the controller parameters $J=$ $f\left(k_{\mathrm{P}}, k_{\mathrm{I}}, k_{\mathrm{D}}\right)$. The requirement for best drift suppression is that $J$ is as small as possible ( $J \stackrel{!}{=} \min )$. For a quantitative evaluation of this requirement and to get reasonable analytical
Table 9. Performance index from Eq. 27 for drift suppression for the three controller types

\begin{tabular}{ll}
\hline & Performance index $J$ \\
\hline $\mathrm{P}$ & $\frac{1}{2 K^{*} k_{\mathrm{p}} \delta\left(1+K^{*} k_{\mathrm{P}}\right)}$ \\
$\mathrm{PI}$ & $\frac{1}{2 K^{*} k_{\mathrm{p}} \delta\left(1+K^{*} k_{\mathrm{P}}\right)+2 K^{*} k_{\mathrm{I}}\left(1+K^{*} k_{\mathrm{P}}\right)}$ \\
$\mathrm{PID}$ & $\frac{1}{2 K^{*} k_{\mathrm{p}} \delta\left(1+K^{*} k_{\mathrm{P}}\right)+2 K^{*} k_{\mathrm{I}}\left(1+K^{*} k_{\mathrm{P}}\right)+2 K^{* 2} k_{\mathrm{P}} k_{\mathrm{D}} \delta^{2}}$ \\
\hline
\end{tabular}

equations for $J$, the transformation from the time to the frequency domain is reasonable. Since we already proved system stability for all three controller types, we can use Parseval's theorem (Newton et al., 1964) for the accomplishment of the transformation as

$$
\begin{aligned}
J=\int_{0}^{\infty} \Delta x^{2}(t) \mathrm{d} t & =\frac{1}{2 \pi} \int_{-\infty}^{+\infty}|\Delta X(s)|^{2} \mathrm{~d} s \\
& =\frac{1}{2 \pi} \int_{-\infty}^{+\infty} \Delta X(s) \Delta X(-s) \mathrm{d} s
\end{aligned}
$$

with

$$
\begin{aligned}
\Delta X(s) & =\frac{1}{1+K \frac{s+\delta}{s} V G_{\mathrm{c}}} \cdot \frac{1}{s} \\
& =\frac{k_{0}+k_{1} s+\ldots+k_{r-1} s^{r-1}}{l_{0}+l_{1} s+\ldots+l_{r} s^{r}}, \quad l_{r} \neq 0
\end{aligned}
$$

according to the system structure in Fig. 13 for a unit step input. The explicit general solution of this integral up to $r=$ 10 can be found in Newton et al. (1964). The insertion of the respective controller transfer function into Eq. (28) gives the compensation deviation $\Delta X(s)$, as stated in Table B2. The solution of the integral according to Eq. (27) and Newton et al. (1964), respectively, leads to the quality criteria $J$ for the three controller types as given in Table 9 (cp. Sect. B2). From Table 9 it is obvious that the equation

$\frac{\partial J}{\partial k_{i}}=0$, with $k_{i} \in\left\{k_{\mathrm{P}}, k_{\mathrm{I}}, k_{\mathrm{D}}\right\}$

cannot be explicitly satisfied. No local minimum in dependence of the controller parameters can be found because the first derivatives $\left(\partial J / \partial k_{\mathrm{P}}, \partial J / \partial k_{\mathrm{I}}, \partial J / \partial k_{\mathrm{D}}\right)$ only converge to zero for infinitely large controller parameters $\left(k_{\mathrm{P}}, k_{\mathrm{I}}, k_{\mathrm{D}} \rightarrow\right.$ $\infty)$. This is not in contradiction to the stability requirements and means that the drift suppression capability of the respective controller type is only restricted by practical limitations of the controller parameters. To exemplarily illustrate the drift suppression of the closed-loop sensor for different controller types, the sensor step responses for selected controller parameters as well as the performance indices $J$ as a function of the respective controller parameter are illustrated in Fig. 14. From Fig. 14 (left) it can be observed that when 

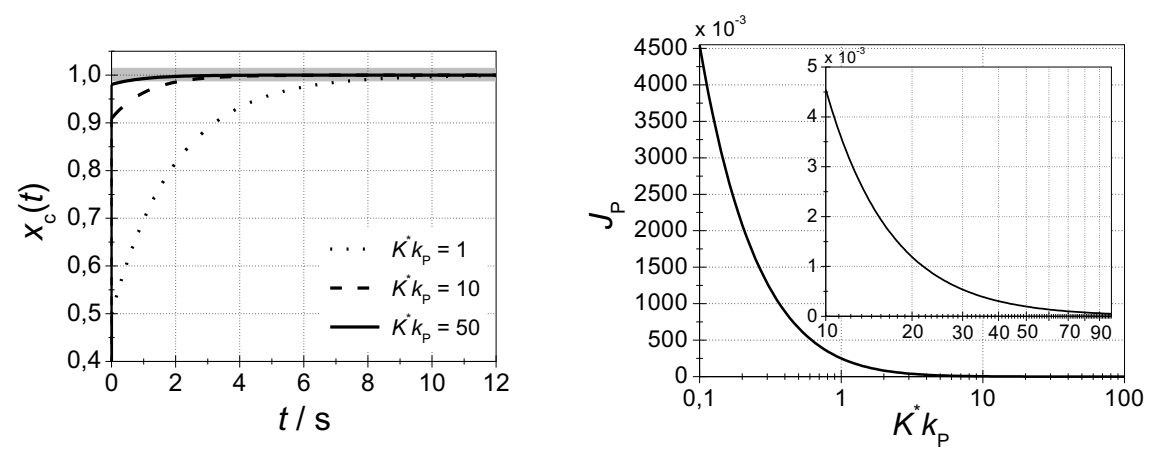

(a)
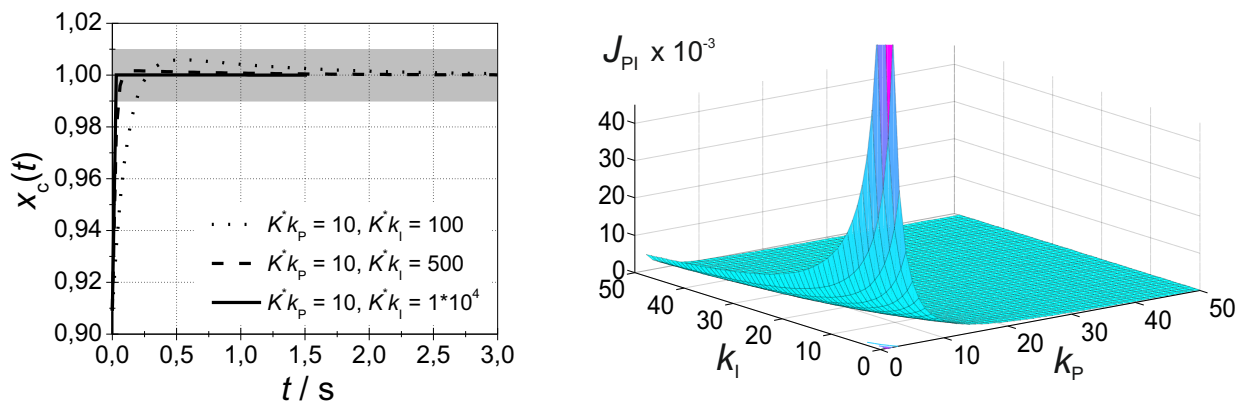

(b)
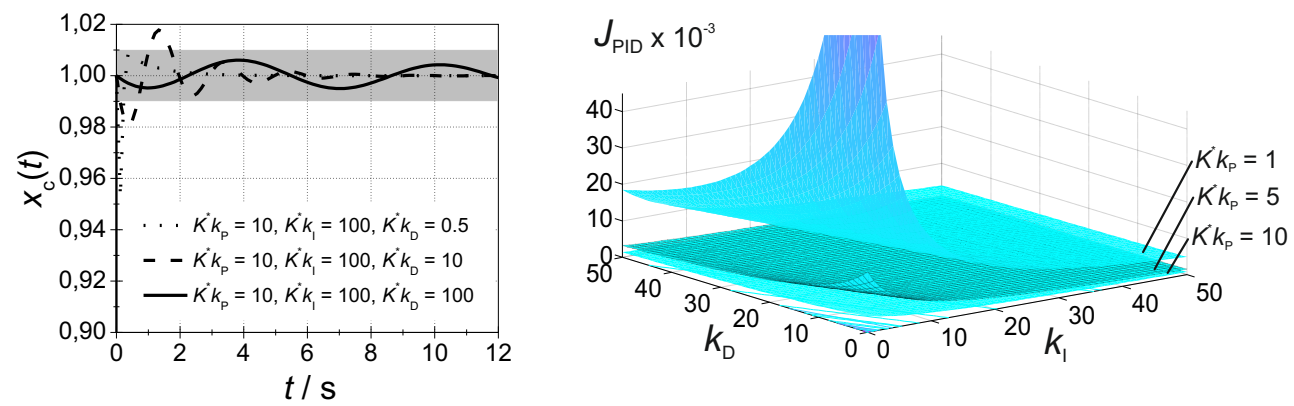

(c)

Figure 14. Step response of the closed-loop sensor for selected controller parameters (left) and ISE performance index as a function of the respective controller parameter (all other parameters are fixed) (right) for a (a) P-controller, (b) PI-controller, and (c) PID-controller. The light gray region indicates a tolerance band of $\pm 1 \%$ of the steady state value. For all graphs a drift rate of $\delta=1 \mathrm{~s}^{-1}$ was considered.

the system experiences an input step, it instantaneously follows this step to a certain extent until it is damped and converges to unity. The higher the respective controller parameter is, the less damping can be observed. Furthermore, the steady state is reached even earlier. Here, reaching the steady state means that the sensor signal sets in into a tolerance band of $\pm 1 \%$ of $x_{\mathrm{c}}(t)=1$ (gray region) and does not leave it anymore. This trend also reflects in the ISE performance indices in Fig. 14 (right). Here, generally $J$ is minimized for increasing controller parameters. Moreover, with increasing controller complexity, $J$ is additionally minimized. However, it can be seen that the P-part has the strongest influence on the quality of drift suppression. It is noteworthy that even a feedback factor of $K^{*} k_{\mathrm{P}}=1$ leads to a complete drift-suppression solely due to the closed-loop sensor structure. However, tuning of the feedback factor leads to a faster accomplishment of the steady state. The I-part shows less influence on the overall drift suppression but can nevertheless be used to improve the time to reach equilibrium. For small $k_{\mathrm{I}}$ an overshoot can be observed due to the zeros of the numerator in the transfer function (Fig. 14b, left). However, for increasing $k_{\mathrm{I}}$, this overshoot decreases. For a PID-controller (Fig. 14c, right), the influence of the I-part is more pronounced than that of the D-part. Both parts show higher influences for small feedback factors. However, for increasing feedback factors, the influence of these parts decreases and becomes negligible for very high feedback factors. The I- and the D-part however influence the signal characteristics. The D-part even leads 
to decaying oscillations within the tolerance band (Fig. 14c, left), which is not an aspired behavior. Thus, we can conclude that the use of a P-controller with high feedback gain can satisfactorily fulfill the demand of drift suppression. A PI-controller is recommendable, if a faster accomplishment of the steady state is aspired, or if the feedback factor cannot be increased due to technical limitations. However, the use of a PID-controller is basically possible but would not be very convenient.

\subsubsection{Non-linearities and hysteresis}

Even if engineering in measurement science aims at a preferably linear system behavior, almost all sensors show nonlinearities to some extent in a certain output signal range. It is known that a non-linearity of a sensor in closed-loop operation is linearized to a certain extent due to the actual operation around a reference working point as

$X_{\text {ref }}-\varepsilon \leq V \cdot X_{\mathrm{o}} \leq X_{\text {ref }}+\varepsilon$,

whereas $\varepsilon$ is kept minimal due to the feedback structure. Hence, in general, a sensor operated by the compensation method will show linearized input-output characteristics compared to the same sensor operated in deflection method. However, this may not be valid for all sensors operated by the compensation method or may just be valid for a limited input signal range. Here, a nonlinear system description can be used because a simple nonlinear model may provide better approximations of the sensor behavior over an expanded range of operation than linear models. Despite the existence of a nonlinear sensor part, the application of linear controllers can be possible. We assume that the sensor dynamics can be decomposed into a static or time-varying nonlinear function $f$ and a linear dynamic part, which is described by transfer function. For this system description, two system models, i.e. the Wiener model and the Hammerstein model, are widely used because of their simplicity and physical meaning. Both models can be used to approximate a wide range of nonlinear dynamic systems (Narendra and Gallman, 1966). The Hammerstein model applied to a nonlinear sensor in closed-loop configuration is shown in Fig. 15. A Wiener model has the reverse system structure where the linear dynamic part is followed by the nonlinear part. Occasionally, combined models with Wiener-Hammerstein or Hammerstein-Wiener structure are also used. The system identification and assignment to one of the above models is an intensive research field, which has to be considered separately and is beyond the scope of this work. However, also for nonlinear sensors in closed-loop configuration, stability has to be assured for all operating conditions. If an existing sensor can be approximated by one of the above models, the stability of the closed-loop system can be achieved if the non-linearity is bounded by a Lipschitz condition or satisfies an appropriate sector condition. The basic idea is to determine the controller parameters which are included in $\tilde{G}_{\mathrm{s}}^{\text {lin }}(s)$

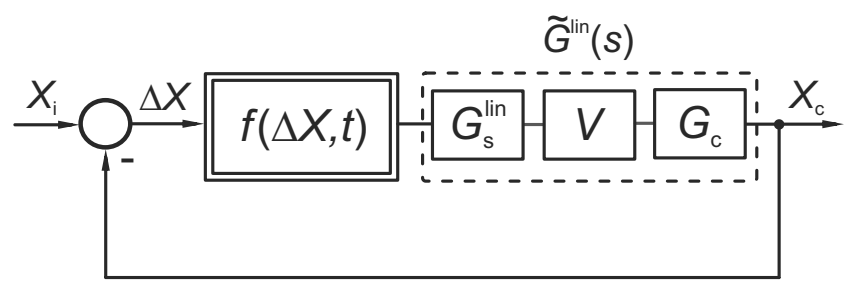

Figure 15. Hammerstein model of a nonlinear sensor in closedloop configuration. The model comprises a static nonlinear part $f$ ahead of a linear dynamic subsystem $\tilde{G}_{\mathrm{s}}^{\text {lin }}$. The linear subsystem is composed of the linear time-invariant part of the sensor transfer function as well as the linear amplifier and controller transfer functions.

in such a way that a specific stability criterion is fulfilled without consideration of the nonlinear part. Various stability criteria do exist for this class of systems, e.g. circle criterion or Popov's criterion (Slotine and Li, 1991; Khalil, 1992).

Sensors exhibiting hysteresis are also nonlinear systems and can thus be described by the models introduced above. However, phenomenologically, the output signal of the core sensor element $G_{\mathrm{s}}$ is kept at a quasi-static state around a reference working point due to the feedback structure (see Eq. 30). Since hysteresis mainly arises from dissipative effects like inner friction inside the core sensor element or large amplitudes (e.g. plastic deformation of a spring element under load), these effects are greatly suppressed by the compensation method when assuming an actuator without hysteresis.

\subsection{Dynamic system behavior}

\subsubsection{Response time}

In general, the sensor response time of any sensor-type is determined by the poles of its transfer function. In the simplest case, only one pole, and thus only one time constant, exists. However, for higher-order transfer functions, several poles do exist. The pole that is located nearest to the imaginary axis in the $\mathrm{LOH}$ is dominant and determines the time to reach equilibrium. The condition for response time reduction is that the dominant pole of the closed-loop system is located in the $\mathrm{LOH}$ relative to the dominant pole of the open-loop sensor

$\mathfrak{R}\left\{s_{\mathrm{p}}^{\text {comp }}\right\}<\mathfrak{R}\left\{s_{\mathrm{p}}^{\text {defl }}\right\}$

as schematically shown in Fig. 16. As apparent from Table 4, the denominator coefficients of all transfer functions depend on the controller parameters. This directly implies that in principle the pole locations can be explicitly set in certain limits by tuning the controller parameters.

\section{Example: $\mathrm{PT}_{1}$-type sensor with P-controller}

The best accessible example to understand the influence of the closed-loop feedback structure on the sensor response 


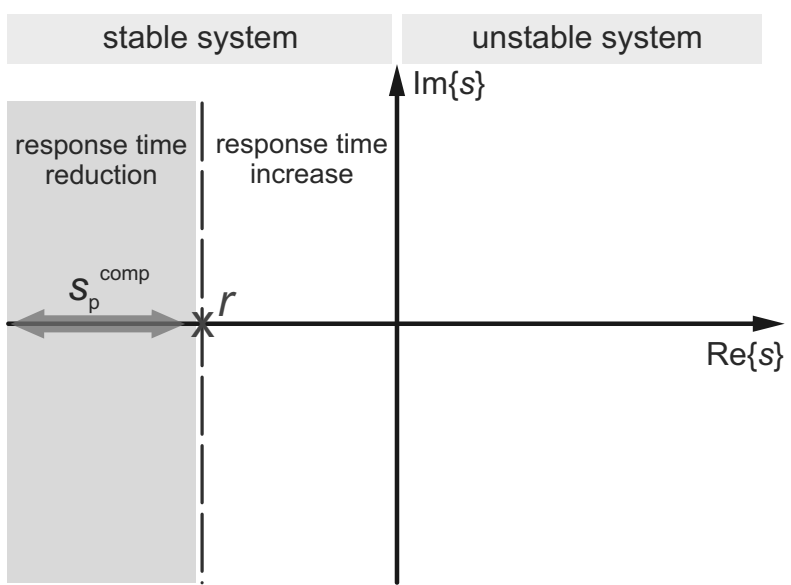

Figure 16. Representation of the complex Gauss plane exhibiting the real part $r$ of the dominant pole $s_{\mathrm{p}}^{\text {defl }}$ of the open-loop sensor and the $\mathrm{LOH}$ relative to this pole (gray region). The dominant pole $s_{\mathrm{p}}^{\text {comp }}$ of the closed-loop sensor has to be located in this half plane to enable a response time reduction according to Eq. (31).

time is the combination of a $\mathrm{PT}_{1}$-type sensor with a $\mathrm{P}$ controller. If one rearranges the respective transfer function from Table 4, one gets

$G_{\mathrm{comp}}(s)=\frac{K^{*} k_{\mathrm{P}}}{1+K^{*} k_{\mathrm{P}}} \cdot \frac{1}{1+s \frac{T}{1+K^{*} k_{\mathrm{P}}}}=\frac{K_{\mathrm{comp}}}{1+s T_{\mathrm{comp}}}$

with

$T_{\text {comp }}=\frac{T}{1+K^{*} k_{\mathrm{P}}}$.

From Eq. (32) one can observe that the closed-loop system is also a first-order system, however with a modified time constant $T_{\text {comp. This time constant depends on the impressed }}$ time constant $T$ of the open-loop sensor itself and inversely on the feedback factor. Thus, $T$ is reduced to $T_{\text {comp }}$ by the factor $\left(1 /\left(K^{*} k_{\mathrm{P}}\right)\right)$ and converges to zero for $K^{*} k_{\mathrm{P}} \rightarrow \infty$. The time-dependent sensor output signal for a unit step input of the open-loop $\mathrm{PT}_{1}$-type sensor as well as the closed-loop sensor comprising a P-controller are shown in Fig. 17a for different feedback factors.

From Fig. 17a, one can observe the influence of different feedback factors on the sensor response time and on the static control deviation, as discussed in Sect. 4.3.1. It is noteworthy that even a unity feedback leads to a minimization of the closed-loop response time of $50 \%$, which can be seen in Fig. 17b. Here, the normalized closed-loop time constant is illustrated as a function of the feedback factor. Fig. 17c schematically illustrates the effect of the feedback structure on the pole location of the closed-loop sensor.

For the combination of more complex controller and sensor types, the transfer functions also become more complex. Hence, for denominator polynomials of increasing order (two or higher), the analytical determination of the poles becomes more demanding. At order two a case differentiation has to be made. For order three a general analytical solution is still possible but not trivial and may not lead to valuable practical conclusions. Therefore, to check the feasibility of a response time reduction and to verify the influence of the different controller types on the response time of the closed-loop system, we use a similar method as already used to proof system stability. This method verifies which requirements have to be fulfilled, so that all poles of the closed-loop transfer function are placed in the $\mathrm{LOH}$ relative to the dominant pole of the respective open-loop transfer function. This directly implies a response time reduction. The steps required to accomplish this are:

- The determination of the dominant poles of the openloop transfer functions $s_{\mathrm{p}}^{\mathrm{defl}}$ and its real parts $r=$ $\mathfrak{R}\left\{s_{\mathrm{p}}^{\mathrm{defl}}\right\}$.

- The redevelopment of the denominator polynomials $D_{\text {comp }}(s)$ of the closed-loop transfer functions at point $s=r$ into the new polynomial $D_{\text {comp }}^{\mathrm{r}}(s)=d_{0}^{\mathrm{r}}+d_{1}^{\mathrm{r}}(s-r)+$ $\ldots+d_{n}^{\mathrm{r}}(s-r)^{n}$ using the Horner scheme.

- The evaluation of the coefficients $d_{0}^{\mathrm{r}} \ldots d_{n}^{\mathrm{r}}$ by application of the Stodola condition and additional application of the Routh criterion (for $n>2$ ) to $D_{\text {comp }}^{\mathrm{r}}$ to evaluate if all poles of the closed-loop transfer functions are placed left of $s=r$ (cp. gray region in Fig. 16).

- The extraction of the controller parameters required to meet these conditions.

A detailed example of this procedure can be found in Appendix C. In Table 10, the dominant time constants of the open-loop sensor as well as the parameter requirements for a response time reduction for all sensor controller combinations are proposed. From this table it can be seen that the application of the compensation method can lead to a decisive response time reduction. For a $\mathrm{PT}_{1}$-type sensor, the requirements are clear and simple. For higher order sensor types, the requirements become more complex. However, in general it can be observed that always one controller parameter only depends on the parameters of the open-loop sensor or has to be simply positive. The remaining controller parameters depend on the respective other controller parameters as well and cannot be chosen individually. This can be exemplarily seen for the closed-loop time constant of a $\mathrm{PT}_{1}$-type sensor in combination with a PI-controller in Fig. 18. Here, the requirements from Table 10 are illustrated. It can be observed that a response time reduction is only possible if the feedback factor amounts to $K^{*} k_{\mathrm{P}}>1$ and if the integral time $T_{\mathrm{I}}$ is smaller than the time constant $T$ of the open-loop sensor. The smaller the integral time is, the better response time reduction can be achieved. However, for every $K^{*} k_{\mathrm{P}}$, a $T_{\mathrm{I}}$ exists where the response time becomes minimal. These minimum points 
Table 10. Dominant time constants $(|1 / r|)$ of the three different sensor types as well as the requirements (parameter range) for a response time reduction of the closed-loop sensor for all considered sensor-controller combinations.

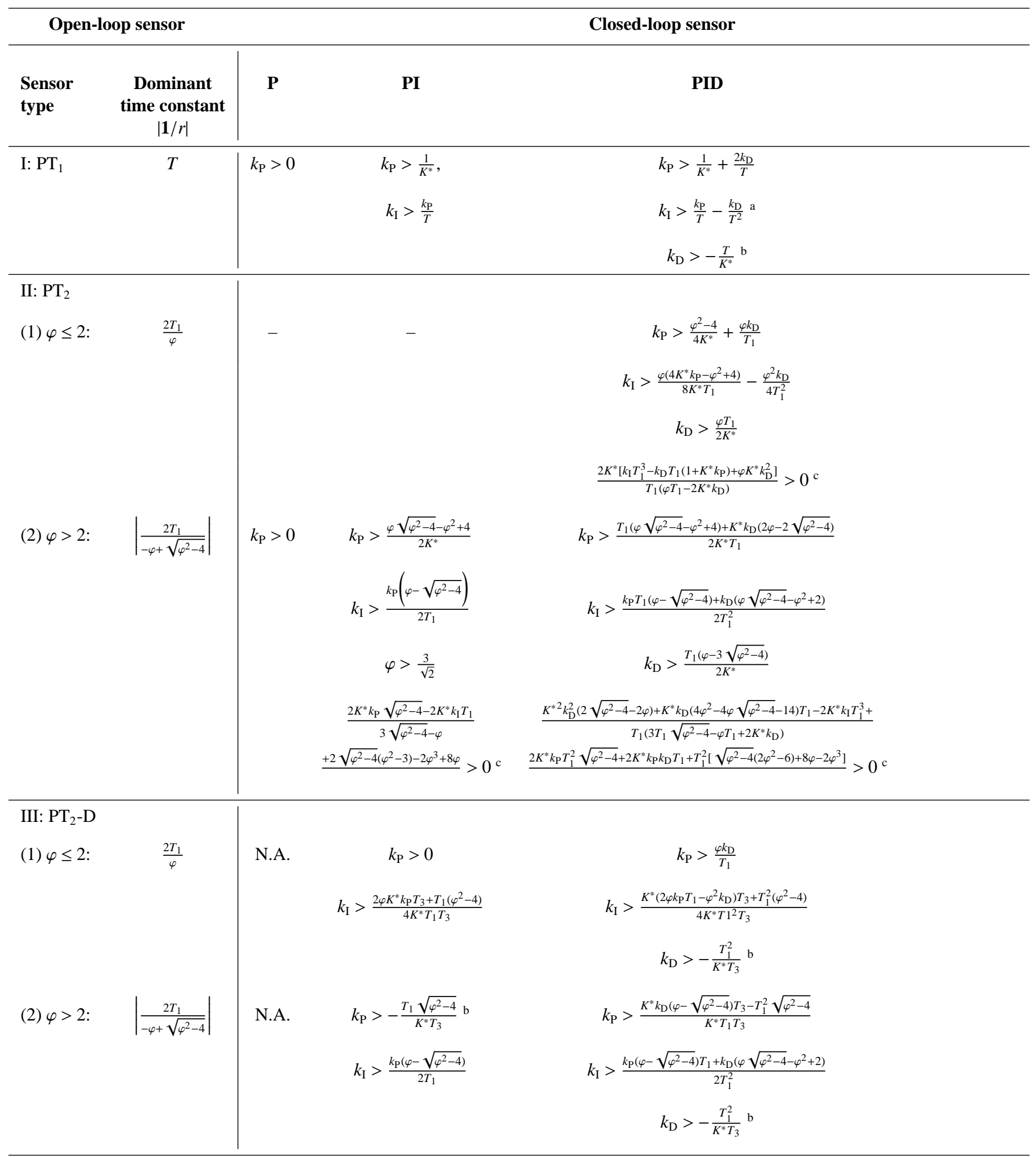

a This condition is only valid for positive $k_{\mathrm{I}}$ due to the stability requirement.

b This condition is automatically fulfilled due to the stability requirement.

${ }^{c}$ This condition results from the application of the Routh criterion. 


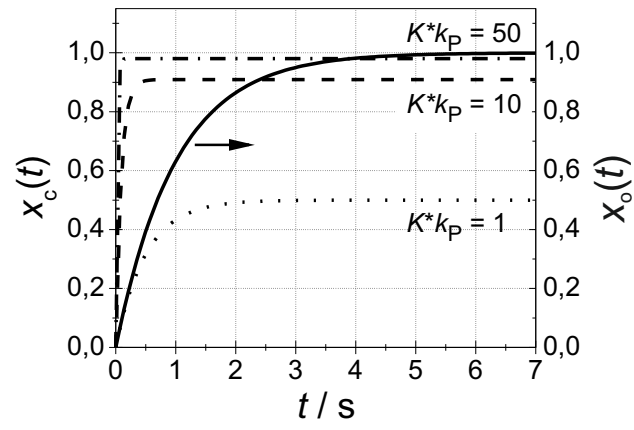

(a)

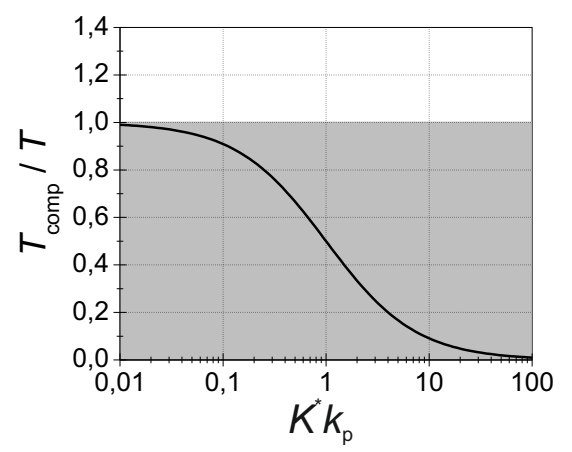

(b)

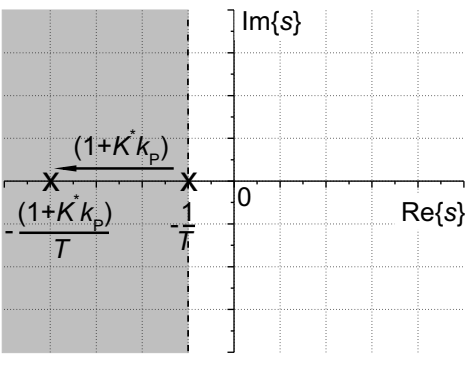

(c)

Figure 17. Influence of compensation method on (a) step response of an open-loop sensor (solid line) for $T=1 \mathrm{~s}$ and a closed-loop sensor (interrupted lines) for different feedback factors; (b) normalized response time of a closed-loop sensor versus feedback factor; (c) schematic illustration of the pole-zero map indicating the pole shift due to the system's feedback structure for compensation. The gray region indicates where a response time reduction is achieved.

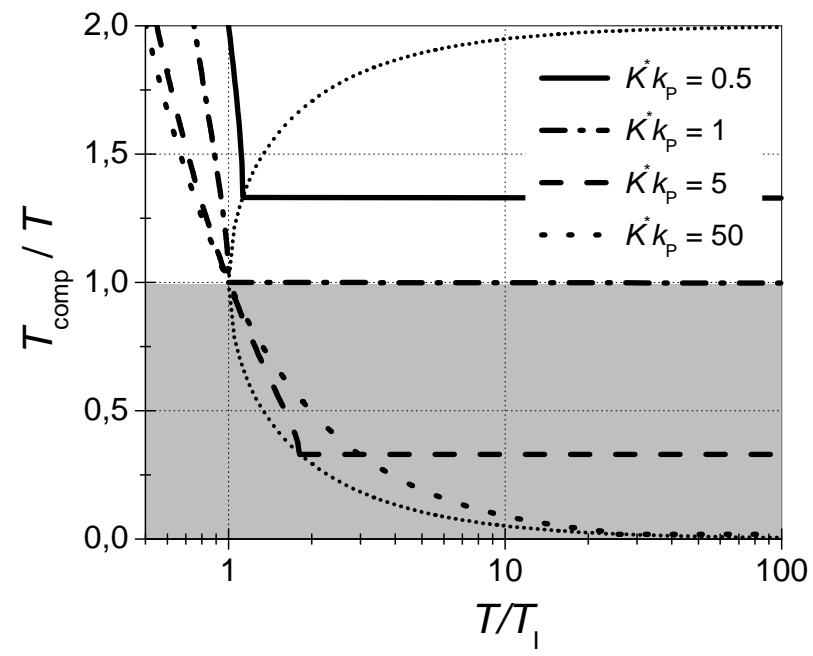

Figure 18. Normalized response time of the closed-loop $\mathrm{PT}_{1}$-type sensor using a PI-controller versus integral time. The gray region indicates where a response time reduction is achieved.

are indicated by the locus (small-dotted line). A further minimization of $T_{\mathrm{I}}$ will only lead to an under-damped oscillatory system but not to a further response time reduction.

For the $\mathrm{PT}_{2}$-type and the $\mathrm{PT}_{2}$-D-type sensor, two different cases according to the pole location were considered: (1) $\varphi=T_{2} / T_{1} \leq 2$ (real double pole or a complex conjugate pole pair), and (2) $\varphi=T_{2} / T_{1}>2$ (two real poles). Note that the combination of $\mathrm{PT}_{2}$-type sensor, which is criticallydamped $(\varphi=2)$ or under-damped $(\varphi<2)$ with a P- or PIcontroller, will never lead to a response time reduction. Only the response time of over-damped systems $(\varphi>2)$ can be reduced using these controller types. However, the application of a PID-controller overcomes this limitation. Due to the dependence of all the specific requirements of all the sensor controller combinations on many parameters, a graphical illustration is not possible. Therefore, the controller parameters enabling a response time reduction have to be calculated for a specific problem. Subsequently, it has to be checked that the obtained parameter range is a subset of the parameter range for a stable minimum-phase system.

\subsubsection{Output signal characteristics}

While the response time is determined by the poles of the transfer function, the zeros of the numerator influence the signal characteristics, i.e. overshoot or undershoot of the step response can occur. With the requirement of a minimumphase system, we already excluded the possibility of an inverse sensor response, i.e. undershoot. However, the location of the zeros with respect to the pole location determines whether the step response shows no maximum, one maximum or even several maxima before settling at the final steady state. For a proper sensor behavior, a step response which attains the final steady state as fast as possible, and hence shows no maximum, is convenient. This is only possible if the transfer function only contains real poles and zeros. The evaluation can be done best by rearranging the general transfer function from Eq. (12) in its pole-zero representation as

$$
\begin{aligned}
G_{\mathrm{comp}}(s) & =\frac{c_{m}}{d_{n}} \frac{\left(s-s_{01}\right) \cdot\left(s-s_{02}\right) \cdot \ldots \cdot\left(s-s_{0 m}\right)}{\left(s-s_{\mathrm{p} 1}\right) \cdot\left(s-s_{\mathrm{p} 2}\right) \cdot \ldots \cdot\left(s-s_{\mathrm{p} n}\right)} \\
& =\frac{c_{m}^{\prime}}{d_{n}^{\prime}} \frac{\left(1+T_{\mathrm{N} 1}\right) \cdot\left(1+T_{\mathrm{N} 2}\right) \cdot \ldots \cdot\left(1+T_{\mathrm{N} m}\right)}{\left(1+T_{\mathrm{D} 1}\right) \cdot\left(1+T_{\mathrm{D} 2}\right) \cdot \ldots \cdot\left(1+T_{\mathrm{D} m}\right)},
\end{aligned}
$$

with

$s_{0 \mu}=-\frac{1}{T_{\mathrm{N} \mu}}, s_{\mathrm{p} v}=-\frac{1}{T_{\mathrm{D} v}}$. 
For further considerations, we assume the time constants to be ordered by size as

$$
\begin{gathered}
T_{\mathrm{N} 1} \geq T_{\mathrm{N} 2} \geq \ldots \geq T_{\mathrm{N} \mu}, \mu=1, \ldots, m, \\
T_{\mathrm{D} 1} \geq T_{\mathrm{D} 2} \geq \ldots \geq T_{\mathrm{D} v}, v=1, \ldots, n .
\end{gathered}
$$

A step response without overshoot (no maximum) is possible if all time constants are real and every numerator time constant $T_{\mathrm{N} \mu}$ is smaller than the corresponding denominator time constant $T_{\mathrm{D} v}$.

Therefore, the controller parameters have to be chosen such that the above requirement is fulfilled. Consequently, a transfer function exhibiting only real poles and no zeros never shows an overshoot of its step response. Note that changing the numerator time constants also changes the denominator time constants, because they depend on each other via the controller parameters and cannot be changed individually.

\section{Conclusions and summary}

Based on a general sensor model, both the deflection and the compensation method have been described and compared in a general systematic fashion. A system-based approach was applied with regard to important figures of merit of sensor technology. In particular, the quasi-static sensor behavior comprising systematic static variations of the sensitivity, long-term drift effects as well as the dynamic sensor behavior, i.e. response time and output signal characteristics, has been described. Three sensor types, i.e. $\mathrm{PT}_{1-}{ }^{-}, \mathrm{PT}_{2-}$, and $\mathrm{PT}_{2^{-}}$ D-type sensors, which cover most of the existing sensors, were analyzed concerning both measurement methods.

In conclusion, for a sensor operated with the deflection method the following applies:

- A static sensitivity change of the sensor due to an external interference quantity directly influences the sensor output signal.

- Quasi-static sensitivity changes, i.e. long-term drift effects, lead to a continuous infinite increase of the output signal, whereas an explicit assignment of the input signal to the output signal is not possible without additional stipulations.

- The response time is impressed by the sensor principle and the sensor material properties, as well as by the underlying physical phenomena, respectively. This directly reflects in the coefficients of the denominator polynomial of the respective transfer function.

- For the transfer functions studied, the output signal characteristics is in direct correlation with the response time of the sensor, and thus just determined by the denominator coefficients.
- For the operation of the sensor, a detailed understanding of the sensor structure or sensor modelling is not necessary. Only sensor calibration is necessary.

- The system complexity is relatively low compared to the compensation method.

For a sensor operated with the compensation method, the following conclusions can be drawn:

- A sensor operated with the compensation method constitutes a feedback system as known from feedback loops in automatic control.

- Different from feedback loops in automatic control, the measuring signal is the compensation quantity, i.e. the output signal of a controller/actuator unit.

- Like for every feedback loop system, system stability plays a decisive role and has to be assured for all sensor operation conditions.

- For the studied closed-loop sensor transfer functions, stability and additional minimum-phase behavior is assured for the set of positive controller parameters.

- Static sensitivity changes of the core sensor element due to an external interference quantity are suppressed by the static gain of the feedback elements. Thus, the influence on the output signal is only marginal, and for some sensor controller combinations even zero.

- Sensors exhibiting long-term drift effects can be stabilized, so that a constant output signal is achieved at the steady state. This is even possible with a simple unity feedback. P- and PI-controllers have been found to be most suitable for drift suppression, whereas a PIDcontroller is less adequate.

- Non-linearities of a sensor in closed-loop operation are linearized to a certain extent due to the actual operation around a reference working point. A sensor operated by the compensation method shows linearized inputoutput characteristics compared to the same sensor operated in deflection method when assuming a linear actuator.

- A sensor exhibiting nonlinear behavior can be operated with the compensation method by using linear standard controllers.

- The poles of the closed-loop sensor transfer function can be tuned by the controller parameters. In principle, the response time can be decisively reduced compared to an open-loop sensor. The requirements for a response time reduction have been calculated for all sensor-controller combinations. However, for a $\mathrm{PT}_{2}$ type sensor with a real double pole or a complex conjugate pole pair, a response time reduction is not possible with a P- or PI-controller. 
- The output signal characteristics depends on the location of the zeros with respect to the location of the poles. However, both zeros and poles are a function of the controller parameters and cannot be set separately.

- To determine the optimized controller parameters, a detailed model of the core sensor element and all possible peripheral devices must exist, which makes the sensor design more demanding.

- Compared to the deflection method, the application of the compensation method results in a higher system complexity due to the necessity of additional devices like a controller and actuator.

\section{Appendix A}

\section{Application of the Routh criterion}

To evaluate the sufficient condition for asymptotic stability of a closed-loop transfer function with denominator degree three or higher, the positive coefficients $d_{v}(v=0,1,2, \ldots, n)$ of the denominator $D_{\text {comp }}(s)$ have to be arranged in the first two lines of the Routh table as follows (Gantmacher, 1959):

\begin{tabular}{l|ccccc}
$\mathrm{n}:$ & $d_{n}$ & $d_{n-2}$ & $d_{n-4}$ & $\cdots$ & 0 \\
(n-1): & $d_{n-1}$ & $d_{n-3}$ & $d_{n-5}$ & $\cdots$ & 0 \\
\hline (n-2): & $e_{n-1}$ & $e_{n-2}$ & $e_{n-3}$ & $\cdots$ & 0 \\
(n-3): & $f_{n-1}$ & $f_{n-2}$ & $f_{n-3}$ & $\cdots$ & 0 \\
$\cdot$ & $\cdot$ & $\cdot$ & $\cdot$ & & \\
$\cdot$ & $\cdot$ & $\cdot$ & $\cdot$ & & \\
$\cdot$ & $\cdot$ & $\cdot$ & $\cdot$ & & \\
$3:$ & $g_{n-1}$ & $g_{n-2}$ & 0 & & \\
$2:$ & $h_{n-1}$ & $h_{n-2}$ & 0 & & \\
1: & $i_{n-1}$ & 0 & & & \\
$0:$ & $j_{n-1}$ & & & &
\end{tabular}

The coefficients $e_{n-1}, e_{n-2}, e_{n-3}, \ldots$ in the third line result from the cross product of lines one and two:

$$
\begin{aligned}
& e_{n-1}=\frac{d_{n-1} d_{n-2}-d_{n} d_{n-3}}{d_{n-1}}, \\
& e_{n-2}=\frac{d_{n-1} d_{n-4}-d_{n} d_{n-5}}{d_{n-1}}, \\
& e_{n-3}=\frac{d_{n-1} d_{n-6}-d_{n} d_{n-7}}{d_{n-1}}, \ldots
\end{aligned}
$$

The calculation of the e-values is carried out until all remaining values are zero. The calculation of the f-values is carried out analogous with the two overlying lines as:

$$
\begin{aligned}
f_{n-1} & =\frac{e_{n-1} d_{n-3}-d_{n-1} e_{n-2}}{e_{n-1}}, \\
f_{n-2} & =\frac{e_{n-1} d_{n-5}-d_{n-1} e_{n-3}}{e_{n-1}}, \\
f_{n-3} & =\frac{e_{n-1} d_{n-7}-d_{n-1} e_{n-4}}{e_{n-1}}, \ldots
\end{aligned}
$$

From these two new lines again further lines can be calculated. Note that the last coefficient in the Routh table matches the absolute term of the denominator polynomial $D_{\text {comp }}$, i.e. $j_{n-1}=d_{0}$.

The Routh criterion is:

The characteristic polynomial $D_{\text {comp }}(s)$ of the closedloop transfer function with positive coefficients $d_{v}$ ( $v=$ $0,1,2, \ldots, n)$ describes an asymptotic stable system, if and only if all coefficients in the first column of the Routh table are positive:

$$
e_{n-1}>0, f_{n-1}>0, \ldots, g_{n-1}>0, h_{n-1}>0, i_{n-1}>0, j_{n-1}>0 .
$$

\section{Example: $\mathrm{PT}_{2}$-type sensor with PID-controller}

As shown in Table 4, the denominator polynomial is $D_{\text {comp }}(s)=T_{1}^{2} s^{3}+\left(T_{2}+K^{*} k_{\mathrm{D}}\right) s^{2}+\left(1+K^{*} k_{\mathrm{P}}\right) s+K^{*} k_{\mathrm{I}}$. Therefore, the complete Routh table reads:

\begin{tabular}{c|ccc}
$3:$ & $T_{1}^{2}$ & $\left(1+K^{*} k_{\mathrm{P}}\right)$ & 0 \\
$2:$ & $\left(T_{2}+K^{*} k_{\mathrm{D}}\right)$ & $K^{*} k_{\mathrm{I}}$ & 0 \\
\hline $1:$ & $\frac{\left(T_{2}+K^{*} k_{\mathrm{D}}\right)\left(1+K^{*} k_{\mathrm{P}}\right)-T_{1}^{2} K^{*} k_{\mathrm{I}}}{T_{2}+K^{*} k_{\mathrm{D}}}$ & 0 & \\
$0:$ & $K^{*} k_{\mathrm{I}}$ & &
\end{tabular}

Therefore, the Routh criterion is:

$T_{1}^{2}>0,\left(T_{2}+K^{*} k_{\mathrm{D}}\right)>0, K^{*} k_{\mathrm{I}}>0,\left(1+K^{*} k_{\mathrm{P}}\right)-\frac{T_{1}^{2} K^{*} k_{\mathrm{I}}}{T_{2}+K^{*} k_{\mathrm{D}}}>0$.

The first three requirements are already fulfilled by the necessary condition that all coefficients of the denominator polynomial have to be positive. Therefore, the third requirement can be regarded as real sufficient condition that must be additionally considered to guarantee system stability.

\section{Appendix B}

\section{Closed-loop sensor with time-dependent parameter variations}

\section{B1 Parameter range for stability and a minimum-phase system for a drift-afflicted sensor}

The parameter range to assure a BIBO-stable and a minimum-phase system for the transfer functions in Table 8 in Sect. 4.3.3 are listed below in Table B1. For the utilization of a PID-controller, the stability requirement arising from the Routh criterion can be rewritten as

$k_{\mathrm{P}}+K^{*} k_{\mathrm{P}}^{2}+K^{*} k_{\mathrm{D}} k_{\mathrm{P}} \delta+\frac{k_{\mathrm{I}}}{\delta}+\frac{K^{*} k_{\mathrm{I}} k_{\mathrm{P}}}{\delta}+K^{*} k_{\mathrm{D}} k_{\mathrm{I}}>K^{*} k_{\mathrm{D}} k_{\mathrm{I}}$.

From this description it can be easily seen that the inequation is fulfilled if the controller parameters are positive. Thus, the closed-loop sensor system - comprising a PID controller - is stable if all controller parameters are positive. 
Table B1. Combined parameter range guaranteeing system stability and a minimum-phase system for a drift-afflicted sensor with linear drift.

\begin{tabular}{ll}
\hline & $\begin{array}{l}\text { Parameter range for a BIBO stable and } \\
\text { minimum-phase system }\end{array}$ \\
\hline $\mathrm{P}$ & $k_{\mathrm{P}}>0$ \\
$\mathrm{PI}$ & $k_{\mathrm{P}} \geq 0, k_{\mathrm{I}}>0$ \\
$\mathrm{PID} \quad$ & $k_{\mathrm{P}}>-\frac{k_{\mathrm{I}}}{\delta}$ and $k_{\mathrm{P}} \geq-k_{\mathrm{D}} \delta, k_{\mathrm{I}}>0, k_{\mathrm{D}}>0$ \\
& $K\left(k_{\mathrm{P}}+\frac{k_{\mathrm{I}}}{\delta}\right)>\frac{K^{2} k_{\mathrm{D}} k_{\mathrm{I}}}{1+K k_{\mathrm{P}}+K k_{\mathrm{D}} \delta}$
\end{tabular}

\section{B2 ISE quality criteria for the different controller types}

Based on the coefficients of the numerator and denominator polynomial of the compensation deviation $\Delta X(s)$, the ISE quality criteria for the different controller types can be calculated according to Table B2.

\section{Appendix C}

\section{Redevelopment of the denominator polynomial}

As a representative practical example of the method of the redevelopment of the denominator polynomial to verify the possibility of a response time reduction, the combination of a $\mathrm{PT}_{2}$-sensor with a PI-controller shall be used. This is accomplished using the steps described in Sect. 4.4.1:

\section{Determination of the dominant pole of the open-loop sensor}

The dominant pole is

$$
s_{\mathrm{p}}^{\mathrm{defl}}=\frac{-T_{2}+\sqrt{T_{2}^{2}-4 T_{1}^{2}}}{2 T_{1}^{2}} .
$$

For the determination of the real part, a case differentiation has to be made:

$$
\begin{aligned}
& \text { (1) } \varphi=\frac{T_{2}}{T_{1}} \leq 2: r_{1}=\mathfrak{R}\left\{s_{\mathrm{p}}^{\mathrm{deff}}\right\}=-\frac{T_{2}}{2 T_{1}^{2}}=\frac{\varphi}{2 T_{1}} \text {, } \\
& \text { (2) } \varphi=\frac{T_{2}}{T_{1}}>2: r_{2}=\mathfrak{R}\left\{s_{\mathrm{p}}^{\mathrm{defl}}\right\}=\frac{-T_{2}+\sqrt{T_{2}^{2}-4 T_{1}^{2}}}{2 T_{1}^{2}} \\
& =\frac{-\varphi+\sqrt{\varphi^{2}-4}}{2 T_{1}} \text {. }
\end{aligned}
$$

2. Redevelopment of the denominator polynomial of the closed-loop sensor at point $s=r$

According to Table 4, the denominator polynomial of the closed-loop transfer function is

$D_{\text {comp }}(s)=T_{1}^{2} s^{3}+T_{2} s^{2}+\left(1+K^{*} k_{\mathrm{P}}\right) s+K^{*} k_{\mathrm{I}}$.

The application of the Horner scheme to Eq. (C4) leads to the general representation of the redeveloped polynomial at point $s=r$ as

$$
\begin{aligned}
D_{\text {comp }}^{\mathrm{r}}(s)= & r+K^{*}\left(r k_{\mathrm{P}}+k_{\mathrm{I}}\right)+r^{3} T_{1}^{2}+r^{2} T_{2} \\
& +\left(2 r T_{2}+3 r^{2} T_{1}^{2}+K^{*} k_{\mathrm{P}}+1\right)(s-r) \\
& +\left(T_{2}+3 r T_{1}^{2}\right)(s-r)^{2} \\
& +T_{1}^{2}(s-r)^{3} .
\end{aligned}
$$

\section{Evaluation of the polynomial coefficients}

The resulting polynomial coefficients $d_{0}^{\mathrm{r}}, \ldots, d_{3}^{\mathrm{r}}$ of the redeveloped polynomial are now calculated for the two different cases. Furthermore, the Stodola condition $d_{0}^{\mathrm{r}}>0, \ldots, d_{3}^{\mathrm{r}}>0$ is applied to calculate the resulting controller parameters.

(1) $r=r_{1}$ :

$$
\begin{aligned}
d_{0}^{\mathrm{r}_{1}} & =\frac{8 K^{*} k_{\mathrm{I}} T_{1}-4 \varphi K^{*} k_{\mathrm{P}}+\varphi^{3}-4 \varphi}{8 T_{1}}>0 \\
d_{1}^{\mathrm{r}_{1}} & =\frac{4 K^{*} k_{\mathrm{P}}-\varphi^{2}+4}{4}>0 \\
d_{2}^{\mathrm{r}_{1}} & =-\frac{\varphi T_{1}}{2}>0 \\
d_{3}^{\mathrm{r}_{1}} & =T_{1}^{2}>0
\end{aligned}
$$

From the evalutaion of the parameters it can be easily seen that $d_{2}^{\mathrm{r}_{1}}>0$ can never be fulfilled. Thus, the Stodola condition is not satisfied. Consequently, a response time reduction is not possible for this case. Hence, further evaluation of the Routh criterion is not necessary.

(2) $r=r_{2}$ :

$$
\begin{aligned}
& d_{0}^{\mathrm{r}_{2}}=\frac{K^{*}\left(2 k_{\mathrm{I}} T_{1}+k_{\mathrm{P}} \sqrt{\varphi^{2}-4}-\varphi k_{\mathrm{P}}\right)}{2 T_{1}}>0 \\
& d_{1}^{\mathrm{r}_{2}}=\frac{2 K^{*} k_{\mathrm{P}}-\varphi \sqrt{\varphi^{2}-4}+\varphi^{2}-4}{2}>0 \\
& d_{2}^{\mathrm{r}_{2}}=\frac{T_{1}\left(3 \sqrt{\varphi^{2}-4}-\varphi\right)}{2}>0 \\
& d_{3}^{\mathrm{r}_{2}}=T_{1}^{2}>0
\end{aligned}
$$

The Routh criterion according to Appendix A can be written as

$d_{1}^{\mathrm{r}_{2}}-\frac{d_{3}^{\mathrm{r}_{2}} d_{0}^{\mathrm{r}_{2}}}{d_{2}^{\mathrm{r}_{2}}}>0$

and rewritten as

$\frac{-2 K^{*} k_{\mathrm{I}} T_{1}+\sqrt{\varphi^{2}-4}\left(2 K^{*} k_{\mathrm{P}}+2 \varphi^{2}-6\right)-2 \varphi^{3}+8 \varphi}{3 \sqrt{\varphi^{2}-4}-\varphi}>0$. 
Table B2. Compensation deviation $\Delta X(s)$ as a function of the controller parameters for the three controller types and the rules for the calculation of the ISE performance index $J$ according to Newton et al. (1964).

\begin{tabular}{llll}
\hline & $\Delta X(s)$ & & Performance index $J$ \\
\hline $\mathrm{P}$ & $\frac{1}{\left(1+K^{*} k_{\mathrm{P}}\right) s+K^{*} k_{\mathrm{P}} \delta}$ & $\hat{=} \frac{k_{0}}{l_{0}+l_{1} s}$ & $\frac{k_{0}^{2}}{2 l_{0} l_{1}}$ \\
$\mathrm{PI}$ & $\frac{s}{\left(1+K^{*} k_{\mathrm{P}}\right) s^{2}+\left(K^{*} k_{\mathrm{I}}+K^{*} k_{\mathrm{P}} \delta\right) s+K^{*} k_{1} \delta}$ & $\hat{=} \frac{k_{0}+k_{1} s}{l_{0}+l_{1} s+l_{2} s^{2}}$ & $\frac{k_{1}^{2} l_{0}+k_{0}^{2} l_{2}}{2 l_{0} l_{1} l_{2}}$ \\
$\mathrm{PID}$ & $\frac{s}{K^{*} k_{\mathrm{D}} s^{3}+\left(1+K^{*} k_{\mathrm{P}}+K^{*} k_{\mathrm{D}} \delta\right) s^{2}+\left(K^{*} k_{\mathrm{I}}+K^{*} k_{\mathrm{P}} \delta\right) s+K^{*} k_{1} \delta}$ & $\hat{=} \frac{k_{0}+k_{1} s}{l_{0}+l_{1} s+l_{2} s^{2}+l_{3} s^{3}}$ & $\frac{k_{2}^{2} l_{0} l_{1}+\left(k_{1}^{2}-2 k_{0} k_{2}\right) l_{0} l_{3}+k_{0}^{2} l_{2} l_{3}}{2 l_{0} l_{3}\left(l_{1} l_{2}-l_{0} l_{3}\right)}$ \\
\hline
\end{tabular}

The condition $T_{1}^{2}>0$ is automatically fulfilled. The remaining inequations can now be solved for $\varphi$ and the respective controller parameters $k_{\mathrm{P}}, k_{\mathrm{I}}$, as stated in Table 10 .

Acknowledgements. The authors would like to thank the German Research Foundation DFG (grant GE 779-24/1) for funding this work.

Edited by: A. Schütze

Reviewed by: two anonymous referees

\section{References}

Abramovitch, D. Y., Andersson, S. B., Pao, L. Y., and Schitter, G.: A Tutorial on the Mechanisms, Dynamics, and Control of Atomic Force Microscopes, in: P. Amer. Contr. Conf., 3488-3502, 2007.

Abramovitch, D. Y., Hoen, S., and Workman, R.: Semi-automatic tuning of PID gains for atomic force microscopes, Asian J. Control, 11, 188-195, 2009.

Bakshi, U. A. and Bakshi, A. V.: Electronic Measurement and Instrumentation, Technical Publications Pune, 3rd Edn., 2009.

Bhushan, B. (Ed.): Nanotribology and Nanomechanics: An Introduction, Springer, 2005.

Bosch GmbH: Bosch Automotive Aftermarket 2009/2010 - Oxygen Sensors, Tech. rep. Robert Bosch GmbH, 2010.

Budzier, H. and Gerlach, G.: Thermal Infrared Sensors: Theory, Optimization and Practice, Wiley, 2010.

Cattell, J. M.: Mental measurement, Philos. Rev., 2, 316-332, 1893.

Che, W. S. and Oh, J. H.: Development of force-balance accelerometer with high accuracy for precision motion measurement, Meas. Sci. Technol., 7, 1001-1011, 1996.

Datta, A., Ho, M.-T., and Bhattacharyya, S. P.: Structure and Synthesis of PID Controllers, Springer, 2000.

Dyer, S. A.: Survey of Instrumentation and Measurement, Wiley, 2001.

Fingerson, L. M. and Freymuth, P.: Fluid Mechanics Measurements, chap. 3, Taylor \& Francis, 2nd Edn., 1996.

Fortina, J., Marte, W., Grüllenberger, R., Hacker, A., Habenbacher, W., Heller, A., Wagner, C., Wacha, P., and Skrabal, F.: Continuous non-invasive blood pressure monitoring using concentrically interlocking control loops, Comput. Biol. Med., 36, 941-957, 2006.

Fraden, J.: Handbook of Modern Sensors: Physics, Designs, and Applications, Springer, 4th Edn., 2010.
Gantmacher, F. R.: Theory of Matrices, Vol. II, Chelsea, New York, 1959.

Gautschi, G.: Piezoelectric Sensorics: Force, Strain, Pressure, Acceleration and Acoustic Emission Sensors, Materials and Amplifiers, Springer, 2002.

Gosh, A. K.: Introduction to Measurements and Instrumentation, Prentice-Hall Of India Pvt. Ltd., 3rd Edn., 2009.

Hoagg, J. B. and Bernstein, D. S.: Nonminimum-phase zeros much to do about nothing, IEEE Contr. Syst. Mag., 27, 45-57, 2007.

Hoffmann, J. (Ed.): Handbuch der Messtechnik, Hanser, 2007.

Hurwitz, A.: Über die Bedingungen, unter welchen eine Gleichung nur Wurzeln mit negativen reellen Teilen besitzt, Math. Ann., 46, 273-284, 1895.

ISO/IEC Guide 99: International vocabulary of metrology - Basic and general concepts and associated terms (VIM), 2007.

Khalil, H. K.: Nonlinear Systems, Prentice Hall, 3rd Edn., 1992.

Kiencke, U. and Eger, R.: Messtechnik: Systemtheorie für Elektrotechniker, Springer, 7th Edn., 2008.

Klaassen, K. B.: Electronic Measurement and Instrumentation, Cambridge University Press, 2002.

Krause, L.: Vergleich von Kompensations- und Ausschlagprinzip am Beispiel der Waegetechnik, Tech. Mess., 71, 643-650, 2004.

Krause, L.: Dynamische Wägetechnik, Wissenschaftlicher Verlag Berlin, 2005.

Lerch, R.: Elektrische Messtechnik: Analoge, digitale und computergestützte Verfahren, Springer, 2011.

Narendra, K. S. and Gallman, P. G.: An iterative method for the identification of nonlinear systems using a Hammerstein model, IEEE T. Automat. Contr., 11, 546-550, 1966.

Newton, G. C., Gould, L. A., and Kaiser, J. F.: Analytical Design of Linear Feedback Control, Wiley, 1964.

NGK, http://www.ngk.de/en/products-technologies/ lambda-sensors/lambda-sensor-technologies/ broadband-lambda-sensor/, last access: 15 April 2012.

Niebuhr, J. and Lindner, G.: Physikalische Messtechnik mit Sensoren, Oldenbourg Industrieverlag, 4th Edn., 2002.

Profos, P. and Pfeifer, T. (Eds.): Handbuch der industriellen Messtechnik, Oldenbourg, 1994.

Schulz, V., Ebert, H., and Gerlach, G.: A novel miniaturizable closed-loop hydrogel-based $\mathrm{pH}$ sensor, in: Sensors, 2011 IEEE, $592-595,2011$.

Shinners, S. M.: Modern Control System Theory and Design, John Wiley \& Sons, 2nd Edn., 1998.

Slotine, J.-J. E. and Li, W.: Applied Nonlinear Control, Prentice Hall, 1991. 
Stewart, J. and Davison, D. E.: On overshoot and nonminimum phase zeros, IEEE T. Automat. Contr., 51, 1378-1382, 2006.

Stodola, A. B.: Über die Regulierung von Turbinen, Schweiz. Bauz., 23, 17-18, 1894.

Stuart-Watson, D. and Tapson, J.: Simple force balance accelerometer/seismometer based on a tuning fork displacement sensor, Rev. Sci. Instrum., 75, 3045-3049, 2004.
Ştefănescu, D. M.: Handbook of Force Transducers: Principles and Components, Springer, 2011.

Tavoularis, S.: Measurement in Fluid Mechanics, Cambridge University Press, 2005.

Visioli, A.: Practical PID Control, Springer, 2006.

Webster, J. G.: The Measurement, Instrumentation and Sensors Handbook, CRC Press, 1999. 